\title{
A composite dynamic mode decomposition analysis of turbulent channel flows
}

\author{
J. Garicano-Mena, ${ }^{\text {a) }}$ B. Li, ${ }^{\text {b) }}$ E. Ferrer, and E. Valero
}

\begin{abstract}
AFFILIATIONS
Departmento de Matemática Aplicada a la Ingeniería Aeroespacial, ETSI Aeronáutica y del Espacio - Universidad Politécnica de Madrid, Plaza del Cardenal Cisneros, 3, E 28040 Madrid, Spain

author to whom correspondence should be addressed: jesus.garicanomena@upm.es

b) Also at: Center for Engineering and Scientific Computation, School of Aeronautics and Astronautics, Zhejiang University, Zhejiang 310027, China.
\end{abstract}

\begin{abstract}
In this contribution, we consider the Dynamic Mode Decomposition (DMD) framework as a purely data-driven tool to investigate both standard and actuated turbulent channel databases via Direct Numerical Simulation (DNS). Both databases have comparable Reynolds number $R e \approx 3600$. The actuation consists in the imposition of a streamwise-varying sinusoidal spanwise velocity at the wall, known to lead to drag reduction. Specifically, a composite-based DMD analysis is conducted, with hybrid snapshots composed by skin friction and Reynolds stresses. A small number of dynamic modes ( 3-9) are found to recover accurately the DNS Reynolds stresses near walls. Moreover, the DMD modes retrieved propagate at a range of phase speeds consistent with those reported in the literature. We conclude that composite DMD is an attractive, purely data-driven tool to study turbulent flows. On the one hand, DMD is helpful to identify features associated with the drag, and on the other hand, it reveals the changes in flow structure when actuation is imposed.
\end{abstract}

Published under license by AIP Publishing. https://doi.org/10.1063/1.5119342

\section{INTRODUCTION}

The multiscale nature of turbulence imposes a series of constrains on the experimental tools and/or numerical techniques employed to describe it. Achieving a well-resolved, statistically independent description of a turbulent flow field requires the usage of dense grids of computational/data-acquisition points and long running times. Modern data-acquisition systems and data-processing techniques and Direct Numerical Simulation (DNS) strategies fulfill the requirements to offer an accurate description. However, the application of such techniques to a single flow realization results in large amounts of data (typically, from gigabytes to terabytes, an authentic treasure trove): performing the data analysis becomes a challenging task.

The question that naturally arises then is, how can one identify those pieces in this trove that are the most relevant for the description of the physical phenomenon investigated?

Feature extraction algorithms assist in the classification of this wealth of data resulting from experiments or DNS computations. Perhaps the most common strategies are the Proper Orthogonal
Decomposition (POD, Refs. 5-8) and the Dynamic Mode Decomposition (DMD, Refs. 9-11) techniques.

Other alternative feature identification strategies exist, e.g., the Empirical Mode Decomposition (EMD), which is closely related to the Huang-Hilbert transform. The EMD has been recently leveraged to discriminate large from small turbulent structures and characterize phenomena like modulation and footprinting in channel flows.

Proper orthogonal decomposition techniques-also known as principal component analysis or Karhunen-Loève decompositionoperate on sequences of snapshots, i.e., either experimental measurements or numerical solutions acquired at successive time instants. An optimal representation of this sequence is provided by the POD method, as features identified by POD are orthogonal to each other. Moreover, when velocity snapshots are considered, these features can be classified in terms of decreasing energy content. These properties justify the application of POD techniques for the analysis of turbulent flows; see, e.g., Ref. 15.

Reduced Order Models (ROMs) for turbulent flows have been presented in the literature by combining proper orthogonal 
decomposition with the Galerkin projection; see, e.g., Refs. 16-20. The Galerkin projection can also be combined with other bases to build low-order models for complex flows. References 21-23 provide low-order models describing transition to turbulence for shear flows. More recently, Ref. 24 introduced a low-order model derived through a two-step Galerkin projection of the Navier-Stokes equations; the resulting model reproduces the periodic generation of streamwise vortices, which in turn are linked to the near-wall dynamics of turbulent boundary layers. ${ }^{25,2}$

Dynamic mode decomposition techniques also operate on flow snapshots. The structures retrieved are not orthogonal to each other but oscillate harmonically at specific frequencies. DMD can be seen from different, complementary perspectives, ${ }^{27}$ e.g., from that of the Koopman analysis ${ }^{9,28,24}$ or from that of global stability analysis; DMD can also be related to discrete Fourier transform analysis ${ }^{3}$ and with spectral POD, as discussed in Refs. 32 and 33. DMD techniques have been applied to many flow problems in both experimental $^{34-38}$ and numerical settings. ${ }^{9,30,40}$ Early DMD applications have been focused mainly on transitional flows; recent investigations leveraging DMD techniques for the analysis of turbulent flows are reported in Refs. 41 and 42.

Great effort has been invested in improving and extending the DMD techniques in Refs. 9 and 10. Recently, Le Clainche and her collaborators $^{13-45}$ introduced a high order version of the DMD algorithm. High Order DMD has been applied to a zero net mass flux jet ${ }^{38}$ and to build reduced order models for vertical-axis turbines. Reference 31 describes an optimized variant of DMD which is less sensitive to noise in the input data. Reference 47 describes a sparsity-promoting variant of DMD, which assists in the selection of a subset of dynamic modes retained. The non-uniform DMD strategy of Ref. 48, standing on compressed-sensing principles, is capable of handling snapshots sampled at varying temporal separation. It also offers the opportunity to reduce the size of the snapshots considered by having recourse to K-means algorithms. By combining both effects, an effective reduction of the computational effort needed to perform the DMD is achieved. Alternatively, Ref. 39 offers a distributed-memory implementation of DMD, based upon a parallelized QR decomposition. This same algorithm was later on applied on composite snapshots-i.e., snapshots formed by considering two or more different magnitudes (velocity components, skin friction, and $\lambda_{2}$ invariant) - to compute laminar-to-turbulent transition in Ref. 40.

Whatever the specific implementation considered, the application of POD/DMD to large turbulent databases is invariably problematic. Since the number of temporal and spatial scales involved is large and many of them are relevant, it is never trivial how to select a few of them that reproduce accurately enough the flow behavior. However, the attempt of reproducing a specific feature or functional (e.g., drag) rather than the complete system behavior may ease the work at hand, as done, e.g., in aerodynamic design.

In this contribution, we consider data-driven feature detection algorithms to analyze standard and controlled turbulent channel flows showing broadband energy spectra. Specifically, we will apply a dynamic mode decomposition technique to turbulent databases generated by the DNS solver described in Ref. 51. The DMD formulation we employ also provides the POD modes of the data sequence as a by-product; see Refs. 10 and 52 . This circumstance allows us to

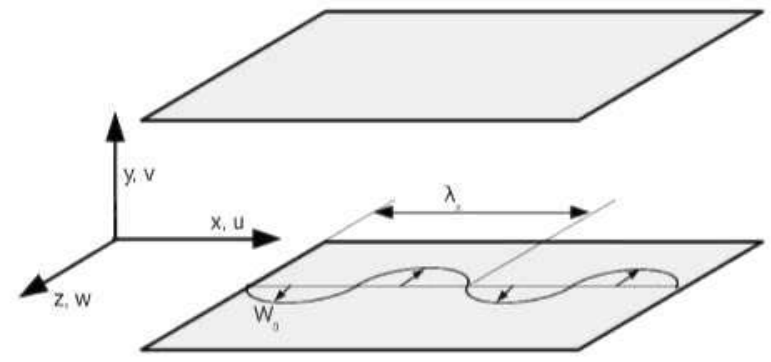

FIG. 1. Domain and system of reference for the channel flow problem. The domain is periodic along $x$ and $z$ directions; bulk flow is along $x$ direction.

acquire further information that, though qualitative in nature, will enrich our DMD analysis.

Encouraged by the promising results in the study of laminarto-turbulent transition in Ref. 40 and supported on the FukagataIwamoto-Kasagi (FIK) ${ }^{53}$ identity, we have attempted to obtain richer information by conducting the POD/DMD analyses on temporal sequences of composite snapshots formed by concatenating Reynolds stresses and wall skin friction. As we shall discuss in Secs. II and III, by using a composite DMD approach, it becomes possible to establish an informed classification of the DMD modes. This classification ultimately allows us to identify a few modes that reconstruct accurately the Reynolds stress distribution responsible of drag generation.

The composite DMD analysis is applied to both standard and actuated channel flows. The actuation considered is the imposition of a spanwise wall velocity that varies sinusoidally with the streamwise coordinate, $w_{\text {watl }} \propto \sin \left(2 \pi x x_{x}\right)$, which is known to lead to drag reduction. See Fig. 1 for the system of reference employed throughout this work.

The long term goal is to reveal whether flow features linked to drag reduction exist and-if that is the case-learn how those structures could be modified to better understand the efficiency of drag reduction strategies.

This contribution is organized as follows: Section II describes the DNS solver employed to generate the turbulent databases and the specific implementations of POD/DMD strategies applied to analyze them. Section 111 discusses the results obtained. Finally, Sec. IV presents the conclusions of our work.

\section{NUMERICAL METHODOLOGY}

\section{A. Databases description}

Feature detection algorithms have been applied to two turbulent channel flows databases generated by the incompressible DNS solver described in Ref. 51. The code follows the paradigm introduced in Ref. 55: it solves for the wall-normal components of velocity $v$ and vorticity $\eta$. This quantities are Fouriertransformed (dealiased using the $3 / 2$ rule) along the homogeneous directions and discretized using explicit compact finite-differences along the wall normal direction. Both the streamwise $u$ and spanwise $w$ velocity components are retrieved using the continuity equation with the relation $\eta=\frac{\partial w}{\partial x}-\frac{\partial u}{\partial z}$. Time integration is accomplished by an explicit third order, low-storage Runge-Kutta 
method, combined with an implicit second-order Crank-Nicolson scheme.

In both the standard and actuated cases considered, the channel walls are planar and the simulations have been conducted under the assumption of constant mass flux. The difference in the databases is the wall boundary condition: in the reference case, the classical no-slip boundary condition has been enforced, whereas in the actuated configuration, a streamwise variation of the spanwise velocity component $w_{\text {wall }}$ is assumed, namely, $w_{\text {wall }}=W_{0}$ $\sin \left(2 \pi x / h_{x}\right)$.

This steady actuation strategy has been investigated in Ref. 54, whereas related purely temporal and spatiotemporal strategies have been considered in Ref. 56 and in Ref. 57, respectively. Reference 58 deals with a related but passive-in-nature drag reduction technique. In every case, the mechanism at play is related to that acting in the temporally oscillating case: provided that the amplitude and wavenumber (and angular pulsation, if applicable) are adequately chosen, the modification induced in the flow field along the transversal direction weakens the viscous near-wall cycle, thus resulting in drag reduction.

The spatial resolution for the standard channel flow is $\Delta x^{+}$ $=6.54$ and $\Delta z^{+}=3.27$ along the homogeneous directions and $\Delta y^{+}$ $\in(0.95,5.18)$; the figures for the actuated flow are $\Delta x^{+}=4.76, \Delta z^{+}$ $=2.38$, and $\Delta y^{+} \in(0.69,3.76)$; the time step enforced in the simulation is in both cases $\Delta t=0.0122$, which corresponds to $\Delta t^{+} \approx 0.1$ for the standard case and to $\Delta t^{+} \approx 0.05$ for the actuated case.

Generating the standard turbulent channel database took $\approx 46 \mathrm{~h}$ on a single core Intel(R) Xeon(R) CPU E5620 at $2.40 \mathrm{GHz}$ with $24 \mathrm{~GB}$ of RAM. The previous solution was used as initial condition to generate the actuated channel database; the computation was run for $\approx 55 \mathrm{~h}$ to shed the transient stage. The code was then run for an additional $\approx 27 \mathrm{~h}$, over which the snapshots for the actuated case were finally stored.

Table I summarizes the characteristics of both databases. Note how the actuation strategy is effective in providing drag reduction, as $R e_{\tau} \approx 200$ for the standard channel, whereas $R e_{\tau} \approx 145$ is observed for the actuated channel flow. ${ }^{54}$

\section{B. Feature detection algorithms: POD and DMD}

We present here a brief summary of the DMD technique, as proposed in Ref. 10. Given a sequence of instantaneous flow fields numbered from 1 to $n_{s}$ (e.g., taking one or all recorded variables), the following data matrix can be constructed:

$$
\mathbf{V}_{1}^{n_{s}}=\left\{\mathbf{v}\left(t_{1}\right), \mathbf{v}\left(t_{2}\right), \ldots, \mathbf{v}\left(t_{n_{s}}\right)\right\},
$$

where the subindex and superindex identify, respectively, the first and last time instants of the sequence. The data is ordered in time and separated by a constant sampling time interval $\Delta t^{s}$ such that $t_{j+1}$ $=t_{j}+\Delta t^{s}$ for all $j=1, \ldots, n_{s}-1$. In the case of linear stability analysis and within the exponential growth region, it is possible to define a linear operator A (i.e., a numerical approximation of the linearized Navier-Stokes operator) such that $\mathbf{v}\left(t_{j+1}\right)=\mathbf{A v}\left(t_{j}\right)$. For nonlinear systems, A represents the Koopman operator. Equation (1) can then be rewritten as a Krylov sequence (see Ref. 60),

$$
\mathbf{v}_{1}^{n_{s}}=\left\{\mathbf{v}\left(t_{1}\right), \mathbf{A v}\left(t_{1}\right), \ldots, \mathbf{A}^{n_{s}-1} \mathbf{v}\left(t_{1}\right)\right\} .
$$

For an ordered sequence, Eq. (2) can be equated to Eq. (1),

$$
\mathbf{A}\left\{\mathbf{v}\left(t_{1}\right), \mathbf{v}\left(t_{2}\right), \ldots, \mathbf{v}\left(t_{n_{s}-1}\right)\right\}=\left\{\mathbf{v}\left(t_{2}\right), \mathbf{v}\left(t_{3}\right), \ldots, \mathbf{v}\left(t_{n_{s}}\right)\right\},
$$

which can alternatively be written in matrix form as

$$
\mathbf{A V}_{1}^{n_{s}-1}=\mathbf{V}_{2}^{n_{s}} \text {. }
$$

Next, the Singular Value Decomposition (SVD) of the matrix $\mathbf{V}_{1}^{n_{z}-1}=\mathbf{U} \mathbf{\Sigma} \mathbf{W}^{H}$ is obtained; the superscript $H$ denotes conjugate transposition. Matrix $\Sigma$ is a diagonal matrix with entries $\sigma_{i}$ the singular values. The left singular vectors-the columns of $\mathrm{U}-$ can be related to the POD modes of the input data sequence: ${ }^{52}$ the DMD algorithm of Schmid offers the POD modes as a by-product.

The SVD of the snapshot matrix is then inserted into Eq. (4), which yields $\mathbf{A U} \Sigma \mathbf{W}^{H}=\mathbf{V}_{2}^{n_{s}}$. The reduced matrix $\widetilde{\mathbf{A}}=\mathbf{U}^{H} \mathbf{A U}$ associated with the initial system described by $\mathbf{A}$, can be rewritten using the former equality as

$$
\widetilde{\mathbf{A}}=\mathbf{U}^{H} \mathbf{A U}=\mathbf{U}^{H} \mathbf{V}_{2}^{n_{s}} \mathbf{W} \mathbf{\Sigma}^{-1} \text {. }
$$

The reduced matrix $\widetilde{\mathbf{A}}$ is the projection of the matrix $\mathbf{A}$ onto the space contained in $\mathbf{U}$, and previously obtained through the SVD operation. The DMD operates under the assumption that the projected matrix $\widetilde{\mathbf{A}}$ conveys most of the information codified into operator A.

Once the reduced matrix $\widetilde{\mathbf{A}}$ has been calculated, the reduced

\begin{tabular}{|c|c|c|c|c|c|c|c|c|c|c|c|}
\hline & $L_{x} / \delta$ & $L_{y} / \delta$ & $L_{z} / \delta$ & $n_{x}$ & $n_{y}$ & $n_{z}$ & $R e_{6}$ & $u_{c}$ & $W_{0} / u_{c}$ & $\lambda_{x} / L_{x}$ & $u_{\tau}$ \\
\hline Reference & \multirow{3}{*}{$2 \pi$} & \multirow{3}{*}{2} & \multirow{3}{*}{$\pi$} & \multirow{3}{*}{192} & \multirow{3}{*}{129} & \multirow{3}{*}{192} & 3678.7 & 0.7733 & $\ldots$ & $\ldots$ & 0.04198 \\
\hline & & & & & & & & & & & \\
\hline \multirow[t]{2}{*}{ Actuated } & & & & & & & 3721.0 & 0.7824 & 0.5 & $1 / 2$ & 0.03077 \\
\hline & \multicolumn{3}{|c|}{ Forcing } & \multicolumn{4}{|c|}{ Snapshots stored $n_{s}$} & $\Delta t^{s}$ & \multicolumn{2}{|c|}{ Memory (GB) } & Time (h) \\
\hline Reference & \multirow{3}{*}{\multicolumn{3}{|c|}{ Constant flow rate }} & \multirow{3}{*}{\multicolumn{4}{|c|}{2049}} & & \multirow{3}{*}{\multicolumn{2}{|c|}{380}} & $\approx 46$ \\
\hline & & & & & & & & 0.122 & & & \\
\hline Actuated & & & & & & & & & & & $\approx 27(+55)$ \\
\hline
\end{tabular}
DMD modes $\mathbf{y}_{i}$ can be obtained, as well as the associated eigenvalues $\mu_{i}$ [i.e., growth rates $\Re\left(\mu_{i}\right)$ and frequencies $\Im\left(\mu_{i}\right)$ mapped to the unit circle] of the reduced system by solving the eigenvalue problem

TABLE I. Databases description 
$\widetilde{\mathbf{A}} \mathbf{y}_{i}=\mu_{i} \mathbf{y}_{i}$. The approximated eigenmodes of the matrix $\mathbf{A}$ can then be recovered via a projection onto the original space, using relation $\phi_{i}=\mathbf{U y}_{i}$. Eventually, the growth rates and frequencies in the complex half-plane can be recovered from the eigenvalues as $\lambda_{i}=\log \left(\mu_{i}\right) / \Delta t^{s}$ (do not mistake the $i$ th eigenvalue $\lambda_{i}$ with the $\lambda_{2}$ invariant).

Finally, note that the DMD decomposition allows us to reconstruct the original data sequence as

$$
\mathbf{v}(t)=\sum_{i=1}^{n_{s}-1} \alpha_{i} \phi_{i} e^{\lambda_{i} t}
$$

In this contribution, the amplitudes $\alpha_{i}$ are computed following the formulation in Ref. 47 . That is, the $\alpha_{i}$ 's stem from the minimization problem in the Fröbenius norm:

$$
\min _{\alpha_{f}}\left\|\mathbf{V}_{1}^{n_{s}-1}-\Phi \mathbf{D}_{\alpha} \mathbf{T}\right\|_{F}^{2},
$$

where the columns in matrix $\Phi$ are the dynamic modes $\phi_{i}$, diagonal matrix $\mathbf{D}_{\alpha}$ contains the unknown amplitudes $\alpha_{i}$, and $\mathrm{T}$ is a Vandermonde matrix whose columns are generated by the successive powers of the column vector $\left[\mu_{1}^{k}, \ldots, \mu_{n_{s}-1}^{k}\right]^{T}$, with $k=0, \ldots, n_{s}-1$. Since matrix $U$ is unitary, it does not affect the norm in Eq. (7), and the optimization problem actually solved is

$$
\min _{\alpha_{i}}\left\|\Sigma \mathbf{W}^{H}-\mathbf{Y} \mathbf{D}_{\alpha} \mathbf{T}\right\|_{F}^{2},
$$

with the columns in matrix $\mathbf{Y}$ the eigenvectors $\mathbf{y}_{i}$ of matrix $\widetilde{\mathbf{A}}$.

\section{POD/DMD analysis of the turbulent databases}

The DNS solver provides the flow state at every $\Delta t t^{s}$ time instant. These flow states consist of the complete velocity field and the skin friction at the wall.

In this work, we have considered two main types of DMD analyses: classical DMD analysis-performed on snapshots of instantaneous Reynolds stress distribution, $u^{\prime} v^{\prime}\left(\vec{x}, t_{j}\right)$-and composite DMD analysis performed on snapshots obtained by concatenating instantaneous skin friction at the wall $C_{f}\left(t_{j}\right)$ and Reynolds stress $u^{\prime} v^{\prime}\left(\vec{x}, t_{j}\right)$. The Appendix describes DMD analyses based on other magnitudes. The rationale behind this choice is supported on the link existing between both magnitudes, as the FIK identity confirms

$$
C_{f}=\underbrace{\frac{12}{R e_{b}}}_{\text {Laminar }}+\underbrace{12 \int_{0}^{1} 2(1-y)\left\langle-u^{\prime} v^{\prime}\right\rangle d y}_{\text {Turbulent }} .
$$

In the equation above, operator $\langle 0\rangle$ represents the temporal and spatial (along the homogeneous directions $x$ and $z$ ) averaging, and $u^{\prime}$ and $v^{\prime}$ are the fluctuations around the statistically stationary profiles $\langle U\rangle(y)$ and $\langle V\rangle(y)=0$.

Observe how, in accordance to factor $1-y$ in the integrand of the turbulent contribution, the closer to the wall, the larger the contribution of the Reynolds stresses to the skin friction is. We will come back to this fact in Sec. III.

Finally, note the upper limit in expansion Eq. (6): if this expansion is truncated-i.e., if a smaller number $n_{r}<n_{S}-1$ is considered-a reduced order model for the process is obtained. Different criteria exist to discriminate which dynamic modes are to be retained into the expansion in Eq. (6), e.g., the cardinalitypenalization-based criterion introduced in Ref. 47 or the one retaining modes with significative time-integrated contributions in Ref. 61.

In this work, we have considered instead two different and very simple criteria. The first criterion consists in retaining only those modes fulfilling $\frac{\left|\alpha_{i}\right|}{\left|\alpha_{\max }\right|} \geq 10 \%$, where the $\alpha_{i}$ 's are the amplitudes in Eq. (6). The second criterion retains in the expansion those modes that contribute most to the skin friction at the wall. This can be accomplished by defining the quantities $\beta_{i} \equiv\left(\phi_{i} \cdot \mathbf{e}_{C_{f}}\right) \alpha_{i}$. If $\mathbf{e}_{C_{f}}$ is the unit vector along the component of the skin friction, then factor $\left(\phi_{i} \cdot \mathbf{e}_{C_{f}}\right)$ extracts the $C_{f}$-related component from the dynamic mode $\phi_{i}$ obtained in a composite DMD analysis. This second criterion, which we term weighted, exploits the correlation between Reynolds stresses and skin friction. As we shall see in Sec. III C, retaining those modes with $\frac{\left|\beta_{i}\right|}{\left|\beta_{\operatorname{sex}}\right|} \geq 10 \%$ allows us to recover with sufficient accuracy the Reynolds stresses distribution for $n_{r} \ll n_{s}$.

Finally, whatever the upper limit $n_{r}$ taken in expansion Eq. (6) is, note that the Reynolds stress profile extracted from the DMD analysis is obtained as

$$
\left\langle u^{\prime} v^{\prime}\right\rangle^{D M D}(y)=\frac{1}{n_{s} \Delta t^{s}} \sum_{i=1}^{n_{f}} \alpha_{i}\left\langle\phi_{i}-\left(\phi_{i} \cdot \mathbf{e}_{C_{f}}\right) \mathbf{e}_{C_{f}}\right\rangle \int_{0}^{n_{s} \Delta t} e^{\lambda_{i} t} d t .
$$

It is those profiles which, in Sec. III, will be compared against those retrieved directly from the DNS simulation.

\section{RESULTS AND DISCUSSION}

In this section, we first assess the adequacy of the turbulent database definition and identify a subset of temporal snapshots that represents accurately the turbulent flow phenomena at the Reynolds

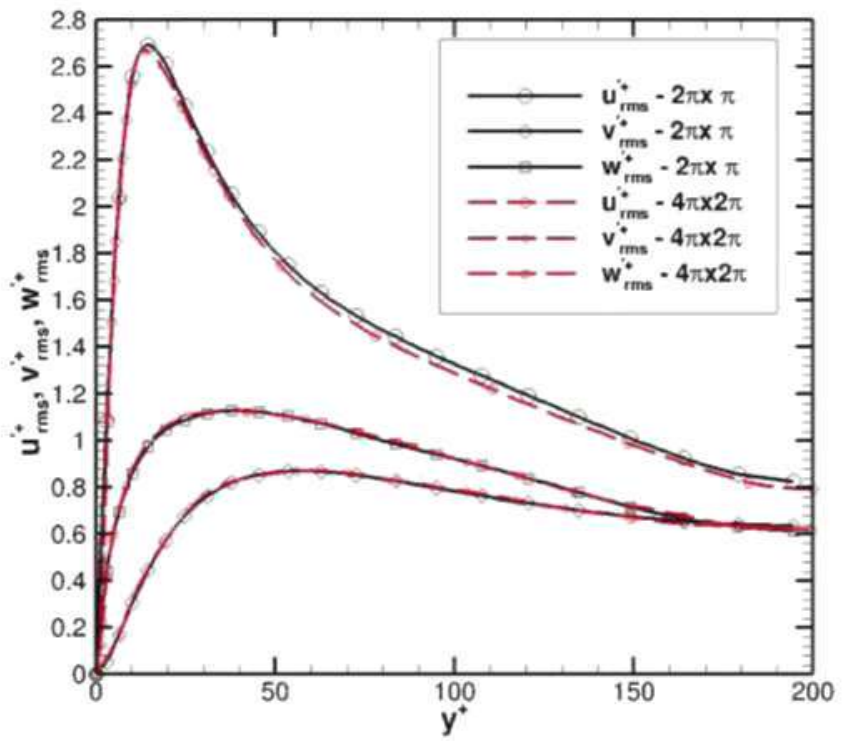

FIG. 2. Standard turbulent channel flow, $R e_{\tau}=200$, and rms of the fluctuations of velocity components. Present DNS results on $2 \pi \times 2 \times \pi$ domain $\left(u_{m m s},-0-\right.$; $V_{\text {rms }},-\backslash$; and $\left.w_{\text {rms }},-\square-\right)$ vs DNS results on $4 \pi \times 2 \times 2 \pi$ domain from Ref, 62 (red, dashed lines). 


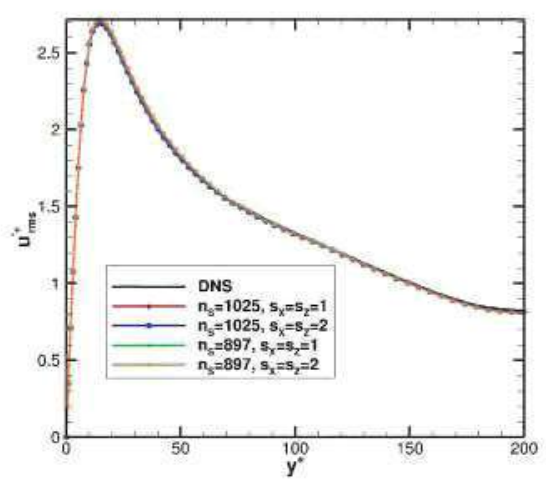

(a)

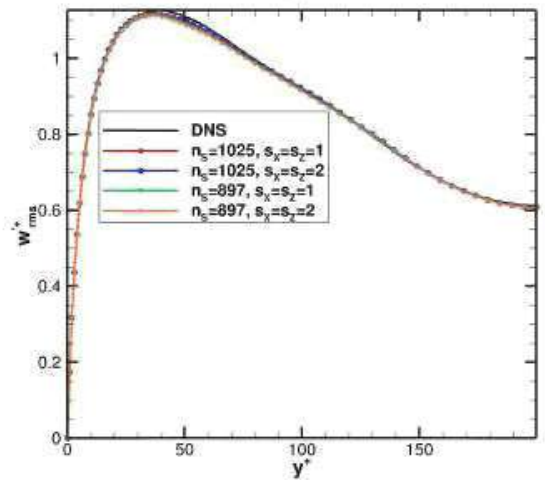

(c)

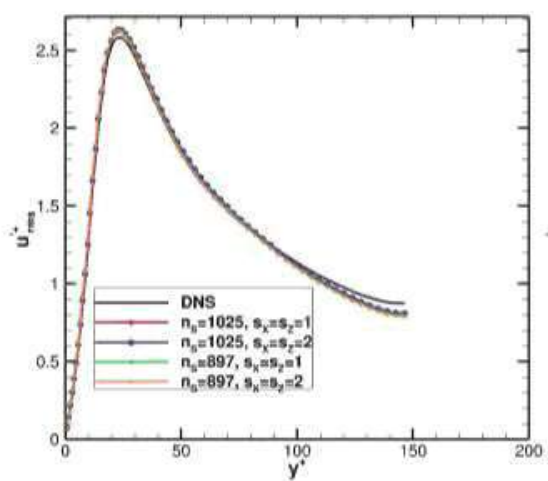

(a)

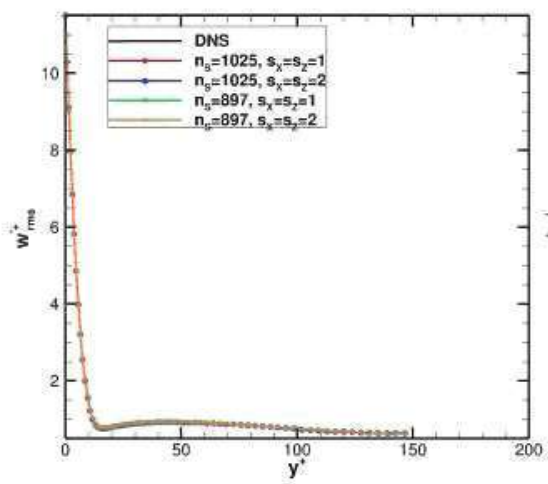

(c)

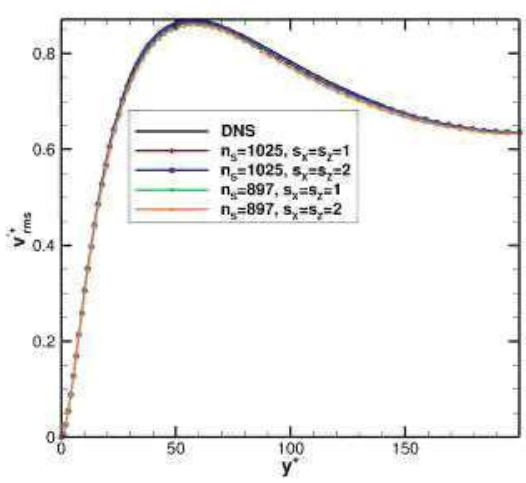

(b)

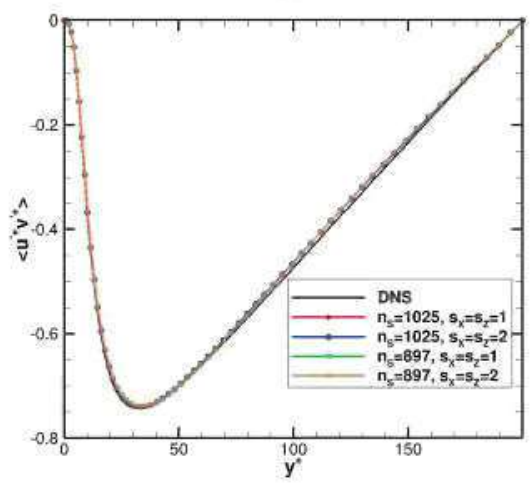

(d)

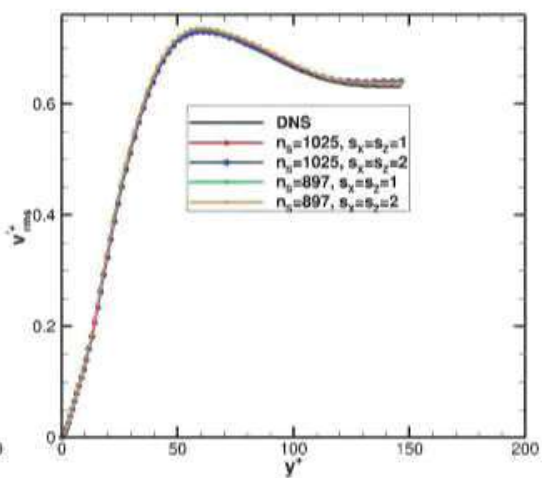

(b)

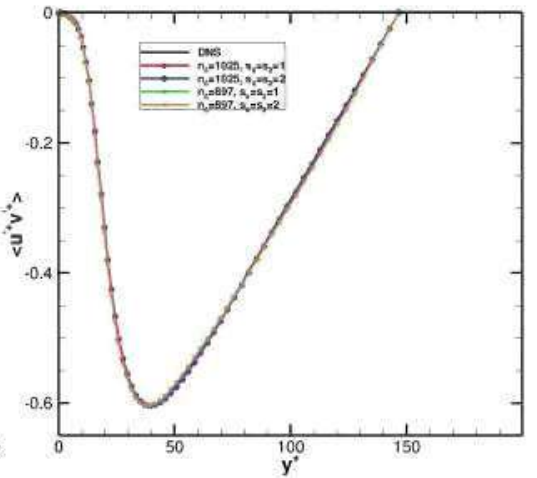

(d)
FIG. 3. Standard channel: data sequence sensitivity to length $n_{s}$ and spatial decimation $s_{x}, s_{z}$ along the homogeneous directions: case $n_{S}$ $=1025, s_{x}=s_{z}=1\left(-\right.$ ); case $n_{s}=$ $1025, s_{x}=s_{z}=2(-\bullet-)$; case $n_{s}=897$, $s_{x}=s_{z}=1(-\mathbf{A}-)$; and case $n_{s}=897$, $s_{x}=s_{z}=2(-\nabla-)$. DNS quantities on $2 \pi \times 2 \times \pi$ domain $(\longrightarrow)$ are included for comparison. (a) $u_{\text {ms }}^{r+}$ (b) $v_{m s}^{\prime+}$ (c) $w_{r m s}^{\prime+}$, and $(d),\left\langle u^{\prime+} v^{\prime+}\right\rangle$.
FIG. 4. Actuated channel: data sequence sensitivity to length $n_{S}$ and spatial decimation $s_{x}, s_{z}$ along the homogeneous directions: case $n_{s}=1025, s_{x}=s_{z}=1$ $(-)$; case $n_{s}=1025, s_{x}=s_{z}=2$ $(-\bullet)$; case $n_{s}=897, s_{x}=s_{z}=1$ $(-\mathbf{\Delta}-)$ and case $n_{s}=897, s_{x}=s_{z}=$ $2(-\boldsymbol{\nabla}-)$. DNS quantities on $2 \pi \times 2$ $\times \pi$ domain $(-$ ) are included for comparison. (a) $u_{m s^{\prime}}^{\prime+}$ (b) $v_{m s,}^{\prime+}$ (c) $w_{m s^{\prime}}^{\prime+}$ and (d) $\left\langle u^{\prime+} v^{r+}\right\rangle$. 


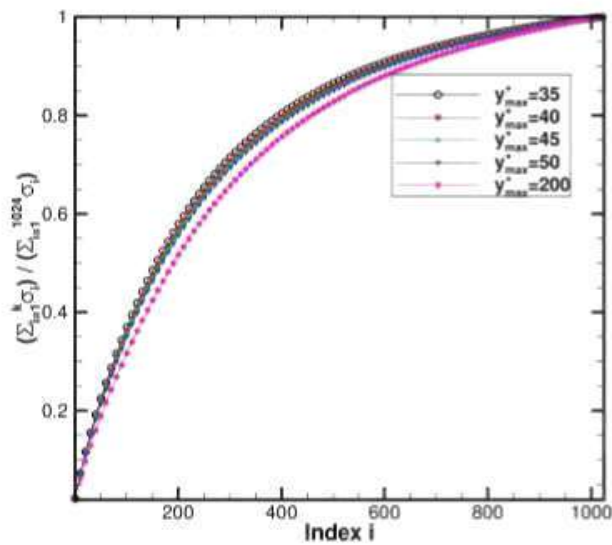

(a)

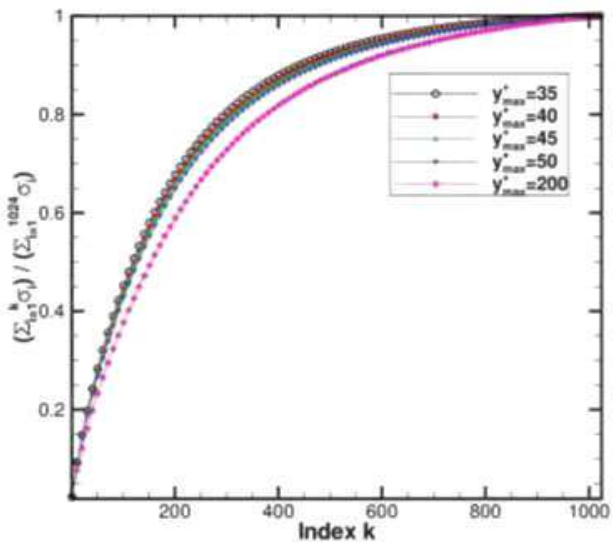

(b)
FIG. 5. POD singular values for standard (a) and actuated channel (b): sensitivity to $y_{\max }^{+}$. stress level (Sec. III A). Next, in Sec. III B, we present the results obtained from the POD analysis of the databases. Finally, we discuss the DMD analysis in Sec. $111 \mathrm{C}$.

\section{A. Data sequence definition and verification}

First, we compare the solution obtained by running the DNS code (on the standard or unactuated case) against the results reported in Ref. 62. The comparison is shown in Fig. 2. Note that both the $x$ and $z$ extents of the computational domain considered in this work are one half shorter that those of the domain considered in Ref. 62; this fact can account for the slight differences appreciable in Fig. 2 .

The second matter of concern is related to the fact that achieving a statistically converged representation of turbulent processes requires averaging on large domains and over long times. However, the feasibility of the QR/SVD decomposition step hinges on the memory available: considering very large domains and/or very long time sequences becomes increasingly costly. The question that naturally arises then is whether the spatial resolution of the snapshots considered and the time span covered by those snapshots is adequate to describe properly the turbulent physical processes we are attempting to analyze.
We address this question in Fig. 3, which shows the secondorder moments obtained from shorter snapshot subsequences ( $n_{s}$ $=897$ and 1025) applying (or not) spatial decimation along the homogeneous directions $x$ and $z$, i.e., retaining every other point (indicated as $s_{x}=s_{z}=2$ ). As visible in Fig. 3, considering spatially decimated snapshots does not impact second-order quantities. Considering shorter sequences does have an effect, albeit very small. Therefore, the data sequence considered in the rest of this work is the one consisting of the first $n_{s}=1025$ snapshots, each of them spatially decimated along both $x$ and $z$ directions $\left(s_{x}=s_{z}=2\right)$. Invoking vertical symmetry, one can also consider a half of the domain. In this manner, the data to be processed by POD/DMD is reduced from the original 2049 snapshots of $n_{x} \times n_{y} \times n_{\bar{z}} \approx 4.76 \times 10^{6}$ points to 1025 snapshots of size $\frac{n_{x}}{s_{x}} \times\left(\right.$ floor $\left.\left(\frac{n_{y}}{2}\right)+1\right) \times \frac{n_{z}}{s_{z}} \approx 5.99 \times 10^{5}$.

Figure 4 describes a similar analysis for the actuated turbulent database; the same observations as for the standard case apply. The data sequence considered is, accordingly, that one formed by the first $n_{s}=1025$ snapshots spatially decimated along both $x$ and $z$ directions, $s_{x}=s_{z}=2$ and considering vertical symmetry.

As argued above, we have conducted POD/DMD analyses on either the standard or the actuated turbulent channel flow databases considering temporal sequences of $n_{s}=1025$ half snapshots, where

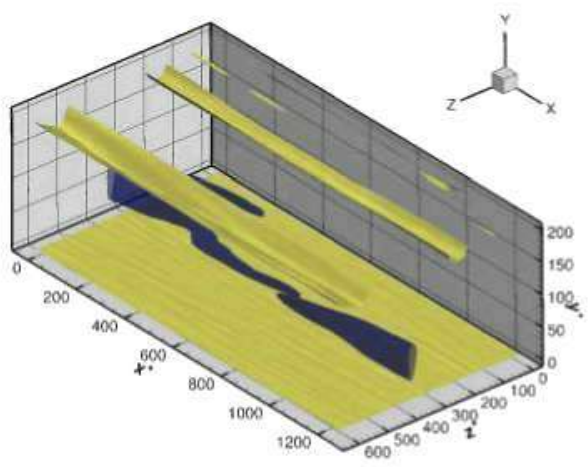

(a) Mode \# 1, standard, $(0.001,2)$

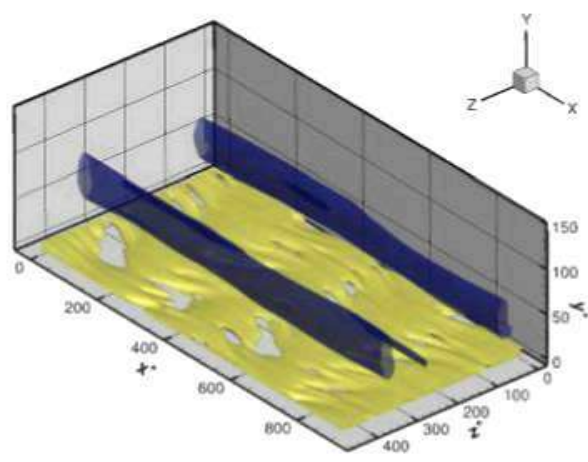

(b) Mode \# 1, actuated, $(0.001,3)$
FIG. 6. POD modes from analysis on $2 \pi$ $\times 1 \times \pi$ domain with $s_{x}=s_{z}=2$, for standard (a) and actuated channel (b). Isosurfaces have been traced at multiples of $u_{\tau}^{2}$, where $u_{\tau}=0.04198$ for the standard and $u_{\tau}=0.03077$ for the actuated channel. The values given in between parentheses specify the aforementioned multiples. 


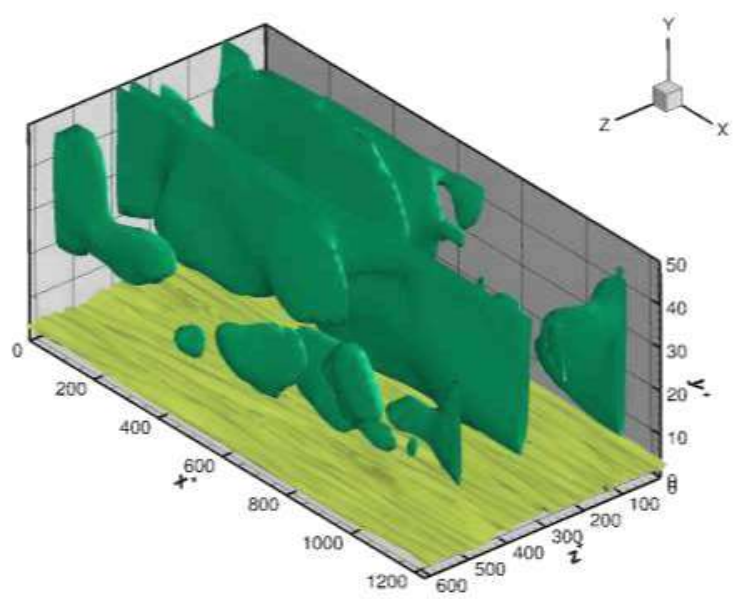

(a) Mode \# 1, standard, $(0.05,1.8)$

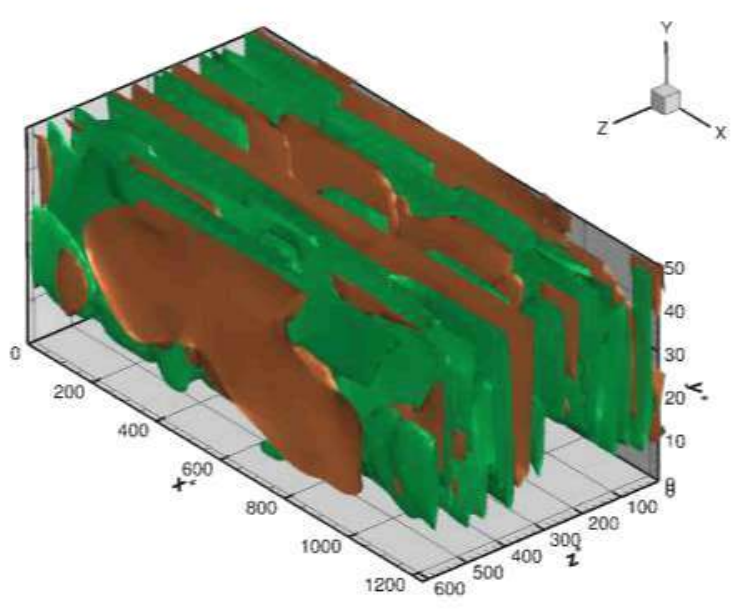

(c) Mode \#2, standard, $(-1,1)$

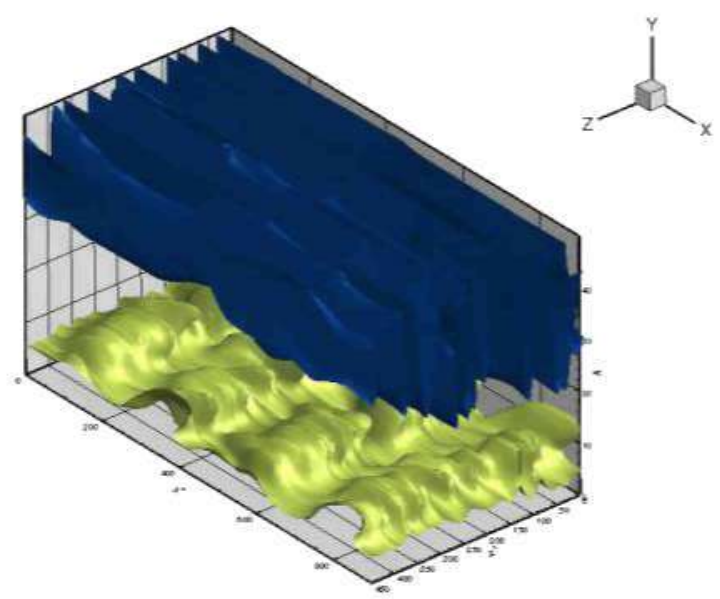

(b) Mode \# 1 , actuated, $(0.05,3)$

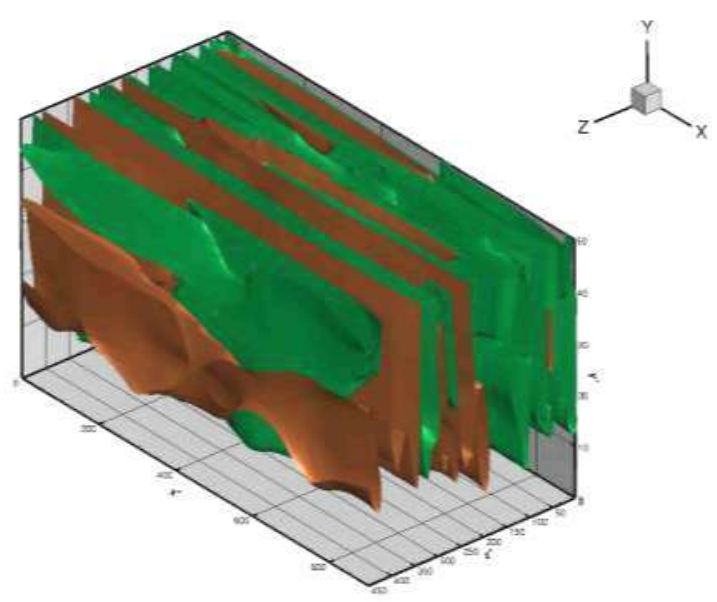

(d) Mode \#2, actuated, $(-1,1)$

FIG. 7. POD modes for standard [(a) and (c)] and actuated channel [(b) and (d)] for $y_{\max }^{+}=50$ (with $s_{x}=s_{z}=2$ ). Isosurfaces have been traced at multiples of $u_{\tau}^{2}$, where $u_{\tau}=$ 0.04198 for the standard and $u_{\tau}=0.03077$ for the actuated channel. The values given in between parentheses specify the aforementioned multiples.

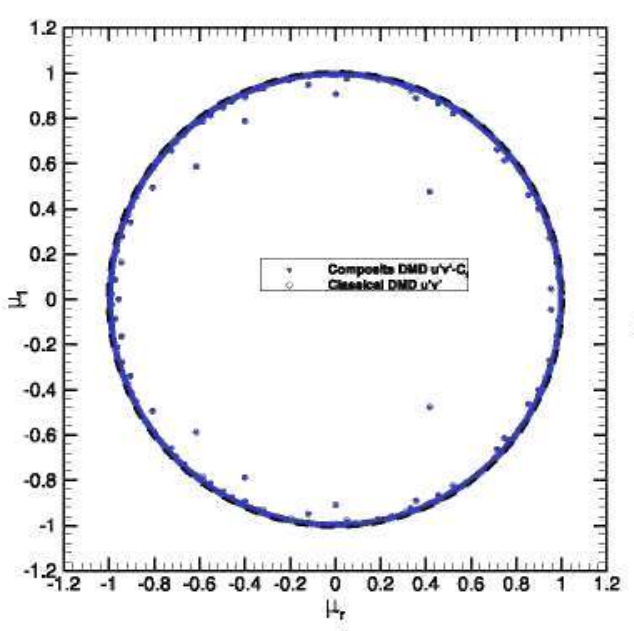

(a)

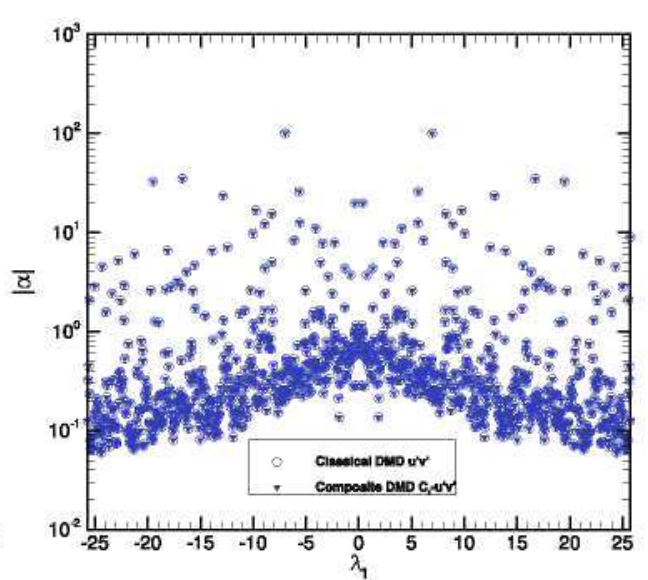

(b)
FIG. 8. Standard channel DMD spectra obtained from analysis based on $u^{\prime} v$ and composite $C_{f}-u^{\prime} v^{\prime}$ snapshots: (a) $\mu$ plane representation with locus $|u|=1$ in dashed line, and (b) amplitude $\left|\alpha_{i}\right|$ vs angular pulsation $\Im\left(\lambda_{i}\right)$. 


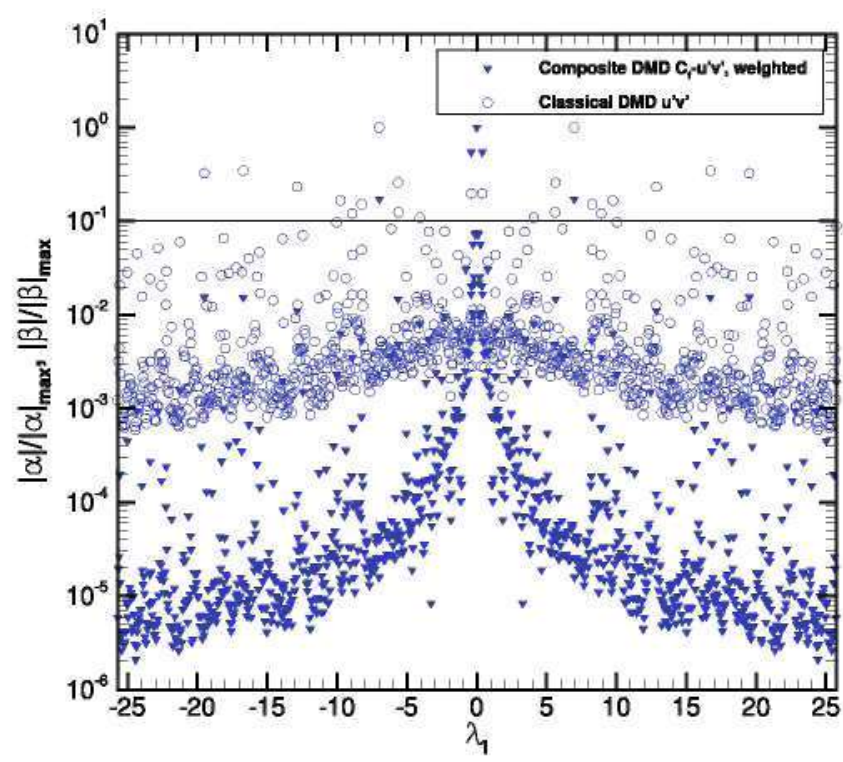

FIG. 9. Standard channel DMD spectra obtained from analysis based on $u^{\prime} v^{\prime}$ and composite $C_{f-u^{\prime}} v^{\prime}$ snapshots: amplitudes $\left|\alpha_{i}\right| /\left|\alpha_{\max }\right|$ and $\left|\beta_{i}\right| /\left|\beta_{\max }\right|$ vs angular pulsation $\Im\left(\lambda_{i}\right)$.

half refers to the region given by $y / h \in[-1,0]$. For reasons to be discussed when describing Fig. 10, we have considered as well the sequences of snapshots in the range $y^{+} \in\left[0, y_{\max }^{+}\right]$for $y_{\max }^{+}=35$, $40,45,50$.

\section{B. POD analysis}

Figure 5 shows the $y_{\max }^{+}$-sensitivity of the cumulative energy associated with the POD decomposition for both the standard [Fig. 5(a)] and the actuated [Fig. 5(b)] channel databases.

Observe how, in both cases, the energy distribution grows in a progressive fashion. Recall that the POD analysis provides $n_{s}-1$ components ordered by decreasing energy content. At the sight of Fig. 5, a distinct mode-index beyond which the supplementary energy contribution is marginal is not apparent. In other words, retrieving a large part of the energy requires most of the POD modes; this is consistent with the broadband character of turbulent flows. This effect can be explained on the account that the smooth energy distribution is consistent with the multiscale nature of the turbulent channel flow: in a turbulent flows, all the scales contribute with energy.

Besides, recall that the POD decomposition used here determines structures that are orthogonal only in space but not necessarily in time. ${ }^{32}$

Note also the difference in the cumulative energy progression between the $y_{\max }^{+}=200$ case (the half-channel) and the $y_{\max }^{+} \leq 50$ analyses. The latter cases are nearly indistinguishable from each other and are distinctly different from the half channel analysis. This suggests that near the wall (i.e., in the inner-layer), the energy is concentrated in fewer modes. We come back to this phenomenon in Sec. III C, when discussing the DMD analysis.

Despite the limitations so far described, the POD analysis is still useful to visualize relevant features of the flow field; see Fig. 6 for POD modes for $y_{\max }^{+}=200$. Figure 7 shows the two first POD modes for both the standard [Figs. 7 (a) and $7(\mathrm{c})$ ] and the actuated [Figs. $7(\mathrm{~b})$ and $7(\mathrm{~d})$ ] channel flows for $y_{\max }^{+}=50$. Note specially the dramatic difference between Figs. 7(a) and 7(b); the effect of the actuation is clearly visible in the neighborhood of the wall.

In conclusion, despite its limitations, the POD information obtained as a by-product of the DMD analysis is nevertheless useful to gain insight into a turbulent flow.

\section{DMD analysis}

Figure 8 compares spectra from both classical (i.e, based on simple $u^{\prime} v^{\prime}$ snapshots) and composite (using $C_{f}-u^{\prime} v^{\prime}$ snapshots) DMD analysis for the unactuated channel flow (the same conclusions are obtained for the actuated case). Both analysis retrieve exactly the same modes $\mu_{i}$; see Fig. 8(a). The majority of those modes lie near the locus $|\mu|=1$, which is in accordance with the statistically stationary nature of the flow. The $|\alpha|$ vs $\Im(\lambda)$ plot in Fig. $8(b)$ shows that also the same amplifications $\alpha_{i}$ are obtained through the classical and the composite DMD analysis. Observe also how most of the modes associated with large $\left|\alpha_{i}\right|$ 's are distributed over the whole range of pulsation frequencies.

According to Fig. 8, classical and composite DMD offer nearly indistinguishable results. It is reasonable to ask then whether composite DMD brings forward any benefit over classical DMD.

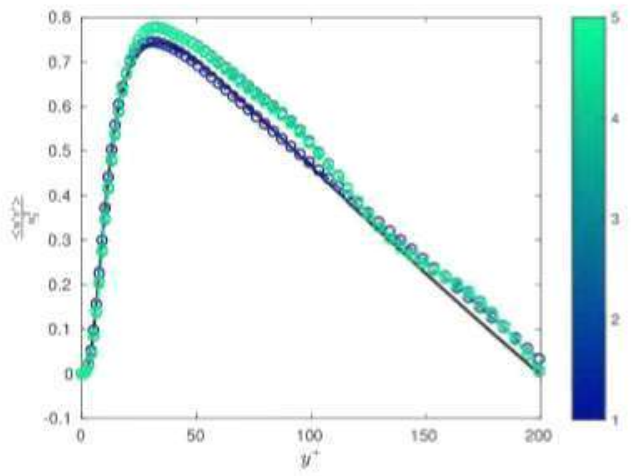

(a)

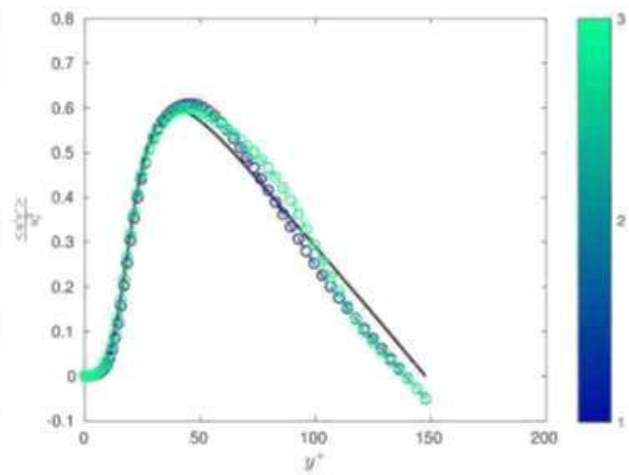

(b)
FIG. 10. DMD-based reconstruction of Reynolds stresses for standard (a) and actuated (b) channels, using modes associated with $|\beta| /\left|\beta_{\max }\right|>10 \%$. Color bar on the right indicates the number of modes considered in the expansion Eq. (10). DNS quantities on $2 \pi \times 2 \times \pi$ domain (-) are included for comparison. 


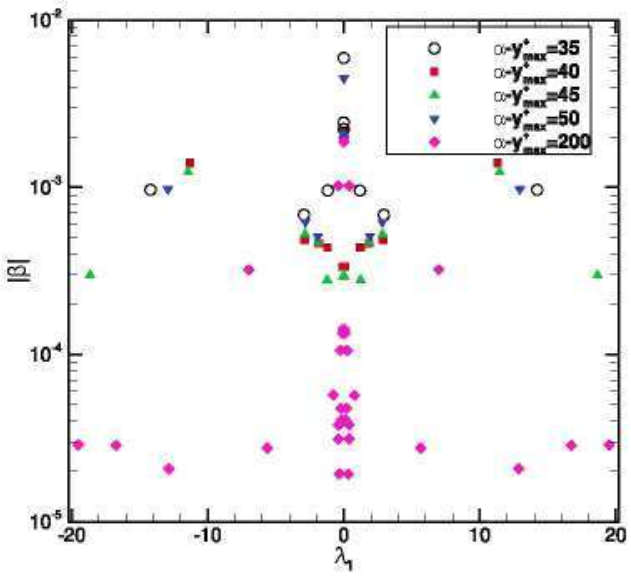

(a)

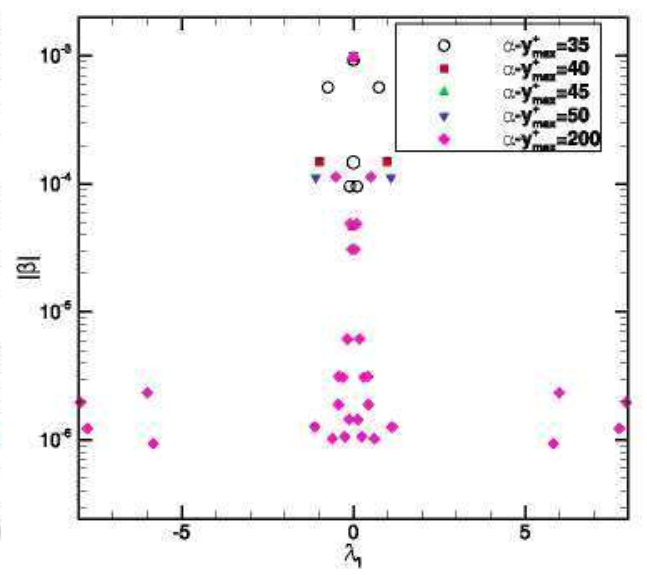

(b)
FIG. 11. DMD spectra for standard (a) and actuated channels (b): sensitivity to $y^{+}$. Only those retained for the reconstruction of $\left\langle u^{\prime} v^{\prime}\right\rangle$ [see Eq. (6)] are shown.
Note however that composite DMD provides additional flexibility with respect to classical DMD: composite DMD allows, for example, to classify dynamic modes according to the $\beta_{i}$ factors introduced in Sec. II C. The physical link between the friction at the wall and Reynolds stresses [recall Eq. (9)] is thus folded into the purely data-based DMD technique.

Previous discussion is illustrated by Fig. 9, which compares classical and weighted composite spectra. First, the most relevant mode appears at $\Im(\lambda)=0$ (i.e., it is a steady mode) in the weighted composite DMD analysis, whereas for the standard analysis, this mode appears at $\Im(\lambda) \approx \pm 7$. Observe also how the weighted composite spectrum presents a segregation of modes in large and small contributions much more distinct than the standard DMD spectrum. This implies that by choosing a fixed $n_{r}<n_{s}-1$, a better description of the skin friction and the Reynolds stresses is obtained with weighted composite than with classical DMD.

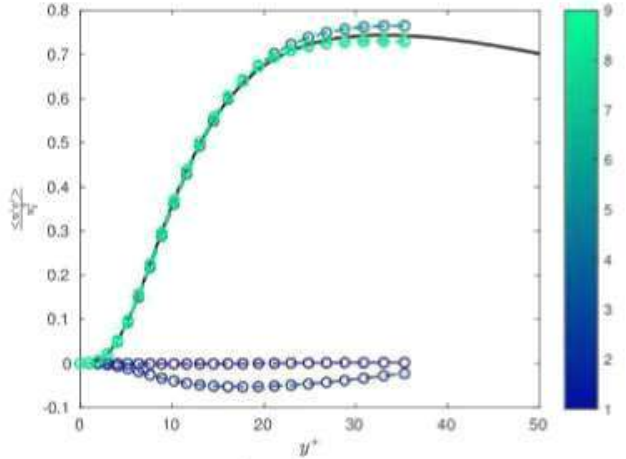

(a) $y_{\text {max }}^{+}=35, n_{r}=9$

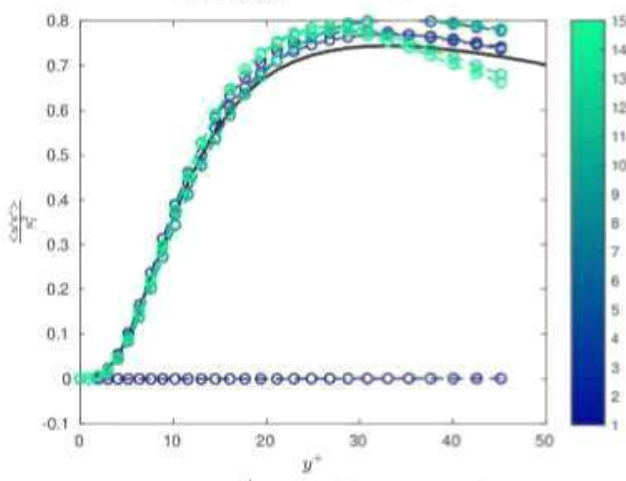

(c) $y_{\max }^{+}=45, n_{r}=15$

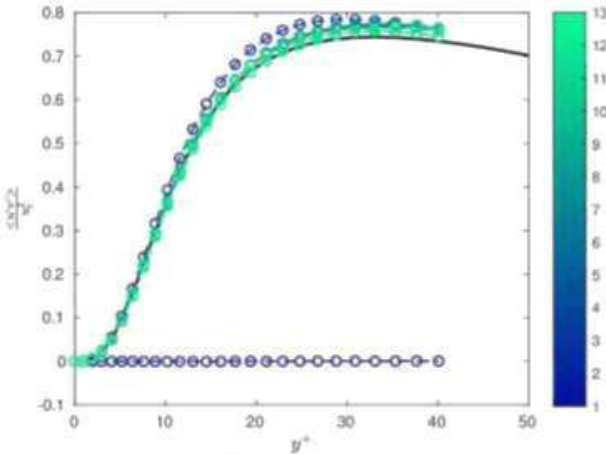

(b) $y_{\max }^{+}=40, n_{r}=13$

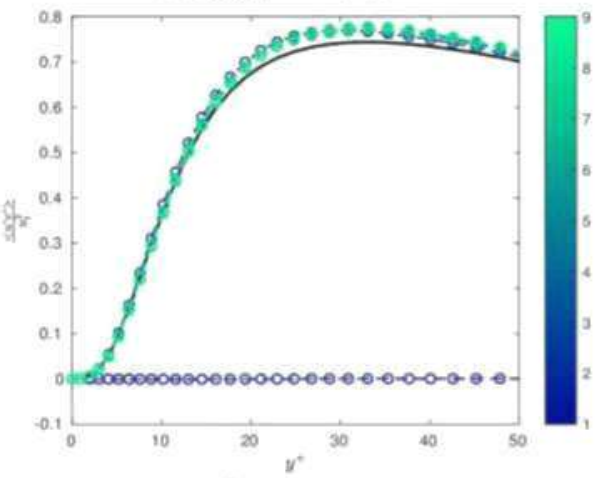

(d) $y_{\text {max }}^{+}=50, n_{r}=9$
FIG. 12. DMD-based reconstruction of Reynolds stresses for standard channel: $y^{*}$-sensitivity. Color bar on the right indicates the number of modes considered in the expansion Eq. (10). DNS quantities on $2 \pi \times 2 \times \pi$ domain $(-)$ are included for comparison. 


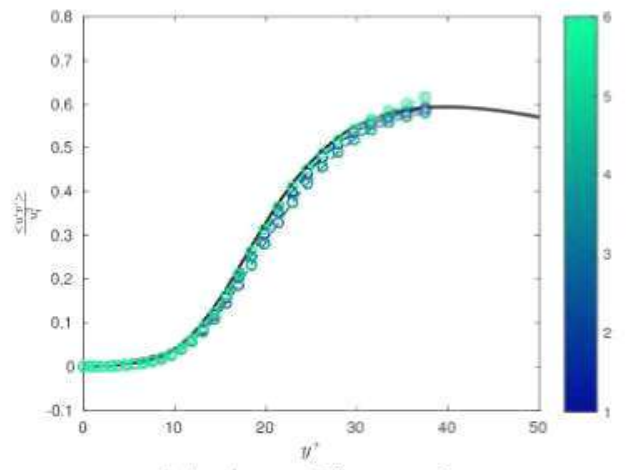

(a) $y_{\max }^{+}=35, n_{r}=6$

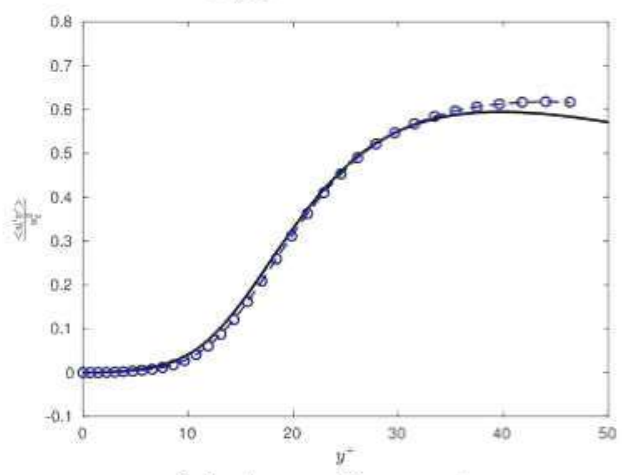

(c) $y_{\max }^{+}=45, n_{r}=1$

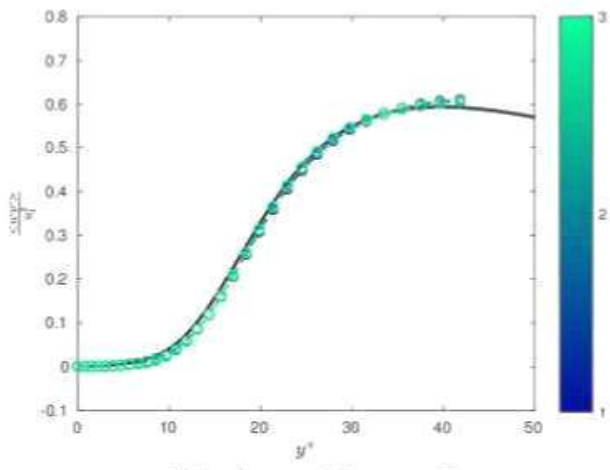

(b) $y_{\max }^{+}=40, n_{r}=3$

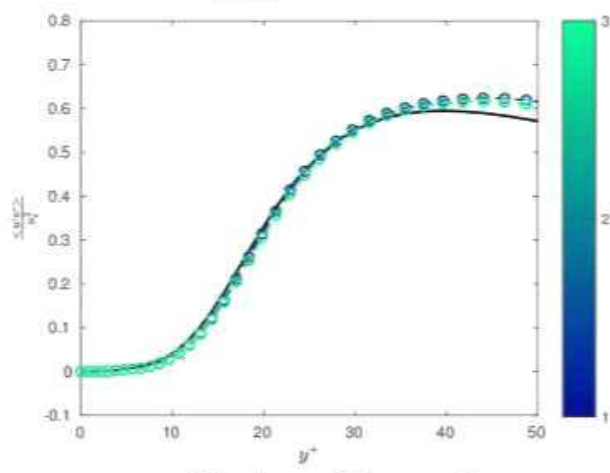

(d) $y_{\max }^{+}=50, n_{r}=3$
FIG. 13. DMD-based reconstruction of Reynolds stresses for standard channel: $y^{+}$-sensitivity. Color bar on the right indicates the number of modes considered in the expansion Eq. (10). DNS quantities on $2 \pi \times 2 \times \pi$ domain $(-)$ are included for comparison.
Hence, from here on, we describe only weighted composite DMD analyses.

Figure 10 shows, for both the standard and the actuated channels, the Reynolds stress corresponding to the reconstruction given by Eq. (6) using a progressively increasing number of retained terms $n_{r}$. The reconstruction with the mode with largest $\left|\beta_{i}\right|$ matches well the Reynolds stress distribution in the range $y^{+} \in[0,100]$.

This behavior can be explained from the viewpoint of Eq. (7). Expansion coefficients $\alpha_{i}^{\prime}$ s are computed through a minimization procedure that guarantees that if $n_{r}=n_{s}-1$, the original data sequence is recovered. However, a truncated DMD expansion, i.e., one with $n_{r}<n_{s}-1$, contrarily to a POD-based expansion, is not necessarily optimal.

The fact that turbulence is an involved multiscale phenomenon complicates further the situation: since there are many relevant features in the flow, it becomes increasingly difficult to represent with a few modes the rich physics of the problem.

Figure 11 shows, for both the standard and the actuated channel flows, the sensitivity of the spectra to the $y_{\max }^{+}$value considered. Spectra for $y_{\max }^{+} \leq 50$ include only those modes that fulfill $\frac{\left|\beta_{i}\right|}{\left|\beta_{\max }\right|} \geq 10 \%$, whereas for the $y_{\max }^{+}=200$ case, the 31 first modes are presented for reference purposes.

The observation above is confirmed by the differences shown by the standard and the actuated flow decompositions. Indeed, one observes from Fig. 11 that less modes with $\frac{\beta_{i}}{\beta_{\operatorname{mix}}} \geq 10 \%$ are found for the actuated than the standard channel. Moreover, note how for the standard channel flow the mean contribution is split up in to three steady modes (see also Fig. 12). This behavior has been described as well in Ref. 43. In the actuated case, a single steady mode is recovered by DMD.

The comparison of Figs. 12 and 13 and Table II confirm this account: i.e., for $y_{\max }^{+}=50$, it is possible to retrieve the correct Reynolds stress distribution; moreover, it is easier to do so for the actuated than for the standard case. However, what would be the reason behind the different behavior of the DMD analysis for the standard and the actuated channels? Since DMD is a data-based feature detection technique, it knows nothing about the physics of the problem. The actuation at the wall introduces a very distinct feature in the flow field; this feature is easily singled out by the optimizer: in a sense, a larger fraction of the physics can be described with a small number of modes.

TABLE II. Error in the reconstruction using Eq. (6).

\begin{tabular}{lccccc}
\hline \hline & \multicolumn{2}{c}{ Standard } & & \multicolumn{2}{c}{ Actuated } \\
\cline { 2 - 3 } \cline { 5 - 5 }$y_{\max }^{+}$ & $L_{2}$ error & $n_{r}$ & & $L_{2}$ error & $n_{r}$ \\
\hline 200 & 0.256 & 5 & 0.116 & 3 \\
50 & 0.179 & 9 & 0.068 & 6 \\
45 & 0.158 & 15 & 0.031 & 3 \\
40 & 0.076 & 13 & & 0.126 & 1 \\
35 & 0.055 & 9 & 0.129 & 3 \\
\hline \hline
\end{tabular}




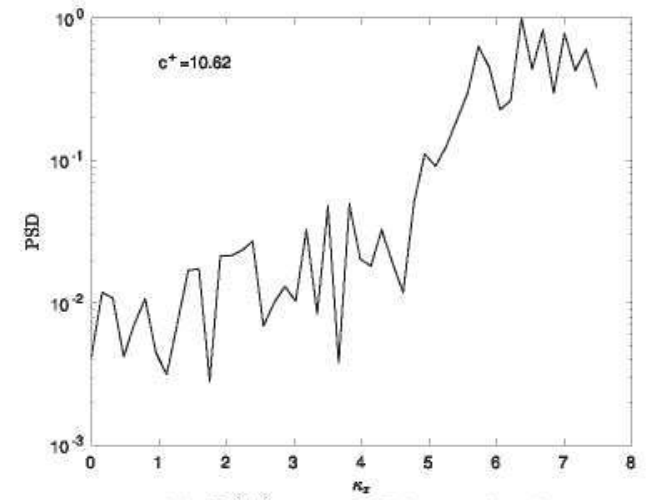

(a) $\mathfrak{I}(\lambda)= \pm 2.85$, standard

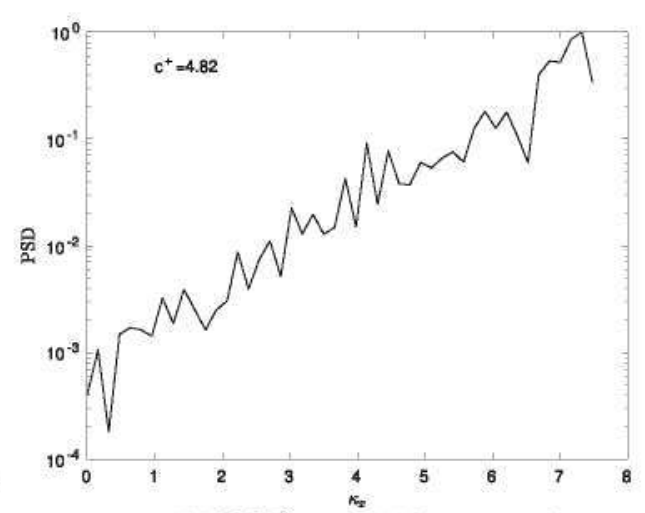

(b) $\mathfrak{I}(\lambda)= \pm 1.10$, actuated
FIG. 14. Streamwise spectra of DMD modes for standard (a) and actuated channels (b) for $y_{\max }^{+}=50$.
In view of the previous argumentation, the relevance of the $y^{+}$sensitivity is understandable: since the near-wall turbulence cycle is sustained even when outer flow turbulent activity is suppressed, by confining the POD/DMD analyses to the near-wall region of the flow field, we are again easing the work of the optimizer. This observation is also in accordance with the $1-y$ term in the FIK relation; see Eq. (9).

Finally, as described in Ref. 41 , it is possible to identify a streamwise propagation speed $c_{x}^{+}$for each of the dynamic modes. Casinelli

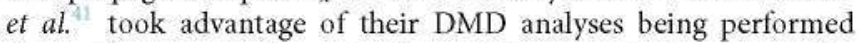
at specific wavenumber pairs $\left(\kappa_{x}, \kappa_{z}\right)$ to compute the streamwise propagation speed $c_{x}^{+}$of the $i$-th mode as

$$
c^{+}=\frac{\Im\left(\lambda_{i}\right)}{\kappa_{x, i}} .
$$

The dynamic modes in our analysis contain, contrarily to those in Ref. 41 , the complete range of spatial wavenumbers. It is nevertheless possible to identify the most relevant streamwise wavenumber by inspecting the spatial Fourier transformation of each of the modes; see Fig. 14.

The most relevant unsteady dynamic mode for the standard channel flow propagates at $c_{x}^{+}=10.62$ (using $\kappa_{x}$ that provides the maximum $P S D$ ), which is very close to the value $c_{x}^{+} \approx 10$ reported in Ref. 65. A value of $c_{x}^{+}=4.82$ is obtained for the actuated case. The values retrieved are robust against variations in $y^{+}$. Moreover, these specific values surface again if a DMD analysis is performed on composite snapshots of $C_{f}$ and $u^{\prime}$.

Finally, let us comment on the computational cost of performing the composite DMD analysis. Table III summarizes the time-in

TABLE III. Time-in seconds-invested in the different phases of the DMD analysis $^{39}$ for $y_{\max }^{+}=200$ case on 48 processors.

\begin{tabular}{lrcrc}
\hline \hline & Standard & $\%$ & Actuated & $\%$ \\
\hline Reading & 5531.52 & 84.90 & 5487.01 & 84.69 \\
QR & 795.23 & 12.21 & 809.98 & 12.50 \\
SVD & 50.11 & 0.769 & 48.95 & 0.750 \\
EIG & 138.81 & 2.13 & 133.22 & 2.06 \\
\hline Total & 6514.97 & 100 & 6479.16 & 100 \\
\hline \hline
\end{tabular}

seconds-invested in the different steps necessary to perform the composite DMD analysis (see Ref. 39) in the $y_{\max }^{+}=200$ case. Note how the most expensive operation is actually loading the database in memory. The next most computationally intensive task is the precursor QR decomposition of the database. The singular value decomposition that provides $\mathbf{U}, \mathbf{\Sigma}$, and $\mathbf{W}$ and the eigendecomposition that offers the Ritz values and the dynamic modes are comparatively much faster. To conclude, observe how there is barely any difference in the time necessary to analyze either the standard or the actuated databases.

\section{CONCLUSIONS}

In this contribution, we have presented a strategy to perform data-driven analyses of turbulent channel flow configurations. Specifically, two turbulent channel flow databases at comparable $R e_{c}$ have been considered. One of them consists of a standard configuration at $R e_{T}=200$, whereas the second one experiences an actuation via the imposition of a streamwise-varying sinusoidal spanwise velocity at the wall. The actuated configuration presents a drag reduction since a diminished $R e_{\tau} \approx 145$ is calculated.

We have conducted composite-based POD/DMD analyses, that is, sequences of snapshots formed by skin friction $C_{f}\left(t_{k}\right)$ and Reynolds stresses $u^{\prime} v^{\prime}\left(\vec{x}, t_{k}\right)$ have been considered. The relevance of the variable choice to assemble the composite snapshots can be justified from the Fukagata-Iwamoto-Kasagi identity.

Either of the databases amounts to slightly less than $400 \mathrm{~GB}$. Since both POD and DMD hinge around a precursor QR decomposition of the snapshots matrix, our first step has been to investigate under which conditions it is possible to retrieve averaged and second-order quantities from reduced views of the database that match closely enough the DNS results. Both reductions in the spatial resolution (spatial decimation, considering half channel) and in the temporal extent have been considered.

Next, a POD-based analysis of both databases has been performed. Contrarily to the initial expectations, the energy distribution grows in a progressive fashion: we have not found a distinct mode-index beyond which supplementary energy contributions are marginal. As POD information is obtained as a by-product of the DMD analysis, POD allows us nevertheless to gain insight into the differences between the standard and the actuated turbulent channels. 
As for the composite DMD analyses and by establishing a link between the $C_{f}$ and the $u^{\prime} v^{\prime}$-related components of the dynamic modes retrieved, we have been able to identify a small number $n_{r}$ of dynamic modes - with $n_{r} / n_{s} \sim \mathscr{Q}\left(10^{-2}\right)$-that allow to reconstruct the Reynolds stresses of the original data sequence. These modes propagate at a range of phase speeds consistent with those originally identified in Refs. 57 and 65 and later retrieved by Casinelli et al.

In summary, in this contribution, we have proposed an avenue to study large turbulent databases through the composite DMD technique. In this work, we have focused on snapshot sequences of Reynolds stresses and skin friction; perhaps other combination of variables provide more accurate or complementary information. This is an avenue to be explored in subsequent work.

\section{ACKNOWLEDCMENTS}

This research has been funded through the NNATAC FP7 initiative (Grant No. PIAP-GA-2012-324298) and by the European Commission Research and Innovation action DRAGY (Grant Agreement No. 690623).

The authors are grateful to Professor M. Quadrio (Politecnico di Milano) for providing the DNS solver. Professor MaríaLuisa Rapún (Universidad Politécnica de Madrid) contributed with valuable comments that improved greatly a first version of this manuscript. We also extend our gratitude to Dr. S. Ghebali for discussions at an early stage of this work.

The authors thankfully acknowledge the computer resources, technical expertise, and assistance provided by the Supercomputing and Visualization Center of Madrid (CeSViMa).

\section{APPENDIX: SUPPLEMENTARY CLASSICAL AND COMPOSITE DMD ANALYSES ON $u^{\prime} v^{\prime}, u^{\prime}$ AND $\lambda_{2}$ MAGNITUDES}

In this appendix, we comment further on the differences observed when performing composite over classical DMD. We illustrate those differences on two auxiliary flow databases. These databases replicate those described in Sec. II A but are computed on a $\pi \times 2 \times \pi / 2$ domain. The specifications are gathered in Table IV. Despite the smaller domain considered for computational convenience, the conclusions attained extend to the larger database.

We have considered a first analysis on the standard turbulent channel auxiliary flow database. Figure 15 shows the reconstructed Reynolds stress profile $\left\langle u^{\prime} v^{\prime}\right\rangle^{D M D}(y)$ computed with Eq. (10). The classical DMD analysis based on $u^{\prime} v^{\prime}\left(\vec{x}, t_{k}\right)$ snapshots results appear in Fig. 15(a). The profile shown is obtained by superposing modes with $\left|\alpha_{i}\right|>10 \%\left(n_{r}=24\right)$; the associated Reynolds stress profile does not reconstruct the DNS computed quantities. However, if a composite DMD analysis based on hybrid $u^{\prime} v^{\prime}\left(\vec{x}, t_{k}\right)-C_{f}\left(t_{k}\right)$ snapshots is considered, the superposition of the $n_{r}=6$ modes with $\left|\beta_{i}\right|>10 \%$ leads to a good approximation of the profile; see Fig. 15(b).

Composite DMD analyses can be performed on snapshots assembled with other quantities as well. Figure 16 presents the results of an analysis performed on snapshots formed by $\lambda_{2}\left(\vec{x}, t_{k}\right)$ fields and $C_{f}\left(t_{k}\right)$. When comparing Figs. $16(\mathrm{a})$ and $16(\mathrm{~b})$, we observe that the superposition of modes associated with largest $\left|\beta_{i}\right|$ provides a flow field characteristic of the near wall region, whereas the superposition of modes associated with largest $\left|\alpha_{i}\right|$ does not.

TABLE IV. Auxiliary databases description.

\begin{tabular}{|c|c|c|c|c|c|c|c|c|c|c|c|}
\hline & $L_{x} / \delta$ & $L_{y} / \delta$ & $L_{z} / \delta$ & $n_{x}$ & $n_{y}$ & $n_{z}$ & $R e_{c}$ & $u_{c}$ & $W_{0} / u_{c}$ & $\lambda_{x} / L_{x}$ & $u_{i}$ \\
\hline Standard & \multirow{3}{*}{$\pi$} & \multirow{3}{*}{2} & \multirow{3}{*}{$\pi / 2$} & \multirow{3}{*}{96} & \multirow{3}{*}{101} & \multirow{3}{*}{96} & 3678.7 & 0.7699 & $\ldots$ & $\ldots$ & 0.04233 \\
\hline & & & & & & & & & & & \\
\hline \multirow[t]{3}{*}{ Actuated } & & & & & & & 3732.7 & 0.7812 & 0.5 & 1 & 0.03136 \\
\hline & \multicolumn{3}{|c|}{ Forcing } & \multicolumn{4}{|c|}{ Snapshots stored $n_{s}$} & $\Delta t^{s}$ & \multicolumn{2}{|c|}{ Memory (GB) } & \\
\hline & \multicolumn{3}{|c|}{ Constant flow rate } & \multicolumn{4}{|c|}{1200} & 0.15625 & \multicolumn{2}{|c|}{32} & \\
\hline
\end{tabular}

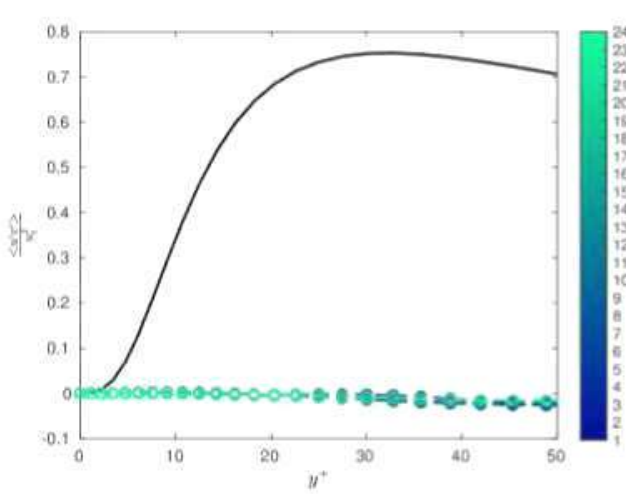

(a) Classical DMD, expansion w/

$$
\frac{\left|\alpha_{i}\right|}{\left|\alpha_{\max }\right|}>10 \%, n_{r}=24 .
$$

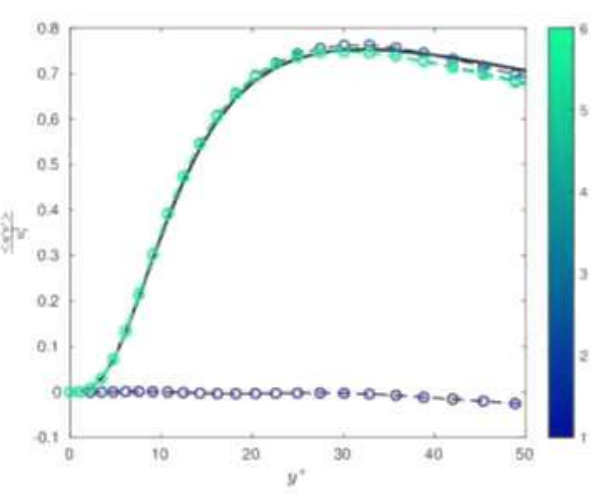

(b) Composite DMD, expansion w/

$$
\frac{\left|\beta_{i}\right|}{\left|\beta_{\max }\right|}>10 \%, n_{r}=6 \text {. }
$$

FIG. 15. DMD analysis of auxiliary turbulent channel flow database at $R e_{\tau}=$ 200 , using $u^{\prime} v^{\prime}\left(\vec{x}, t_{k}\right)$ snapshots, in (a), and composite $u^{\prime} v^{\prime}\left(\vec{x}, t_{k}\right)-C_{f}\left(t_{k}\right)$ snapshots, in (b). Reconstruction of Reynolds stresses with expansion Eq. (10). Color bar on the right indicates the number of modes $n_{r}$ retained. DNS quantities on $\pi \times 2 \times \pi / 2$ domain $(\longrightarrow)$ are included for comparison. 


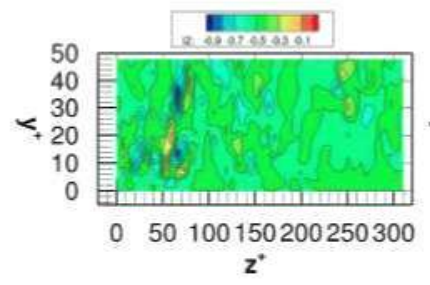

(a) Classical DMD, expansion w/

$$
\frac{|\alpha|}{\left|\alpha_{\text {set }}\right|}>10 \%, n_{r}=30
$$

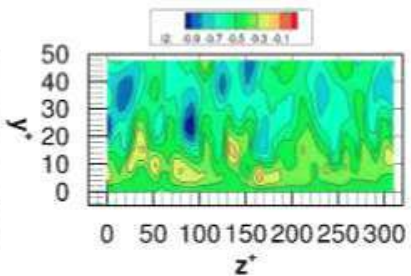

(b) Composite DMD, expansion w/

$$
\frac{\left|\beta_{1}\right|}{\left|\beta_{\operatorname{mar}}\right|}>10 \%, n_{r}=16
$$

FIG. 16. DMD analysis of auxiliary turbulent channel flow database at $R e_{\tau}=$ 200 , using $\lambda_{2}\left(\vec{x}, t_{k}\right)$ snapshots, in Fig. (a), and composite $\lambda_{2}\left(\vec{x}, t_{k}\right)-C_{f}\left(t_{k}\right)$ snapshots, in (b). Reconstructed flow field using Eq. (6), averaged over time and the x-direction.

Another composite DMD analysis has been performed, this time considering snapshots assembled by $u^{\prime}\left(\vec{x}, t_{k}\right)$ and $C_{f}\left(t_{k}\right)$; see Figs. 17-19.

A note on the $u^{\prime}$-based DMD analysis: as discussed inamong others-, ${ }^{41}$ the cyclic regeneration process of near wall structures involves a unstable phase of streak break down. The DMD algorithm captures this behavior (see also Ref. 41), and accordingly, there are eigenvalues $\lambda_{i}$ with a positive real part; see Fig. 17.

The results obtained for the standard channel flow analysis appear in Fig. 18. Specifically, the reconstructed field averaged along time and the $x$-direction shown in Fig. 18(a) suggests the presence of streamwise vortices. The characteristic spanwise spacing is about 100 plus units. Figure 18 (b) shows the instantaneous reconstructed flow [using $n_{r}=5$ in Eq. (6)]. Here again, the characteristic spanwise spacing is around 100 plus units and streak-like features are appreciable (Refs. 25, 41, and 55).

The same analysis has been performed for the auxiliary actuated database. The results from this analysis appear in Fig. 19. Compare Fig. 19(b) against Fig. 18(b): observe how the boundary between alternated low and high speed regions is less pronounced, which is consistent with the reduced $R e_{T}$ of the actuated flow.

In conclusion, composite DMD analysis offers enhanced capabilities with respect to classical DMD. As we have shown, using hybrid snapshots combining $C_{f}\left(t_{k}\right)$ and other flow field [be it $u^{\prime}\left(\vec{x}, t_{k}\right), u^{\prime} v^{\prime}\left(\vec{x}, t_{k}\right)$, or $\left.\lambda_{2}\left(\vec{x}, t_{k}\right)\right]$ allows us to establish an informed classification of the DMD modes. This classification ultimately

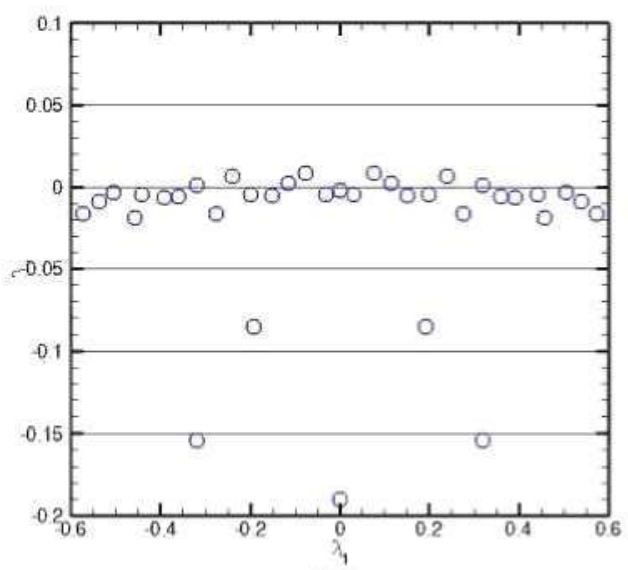

(a)

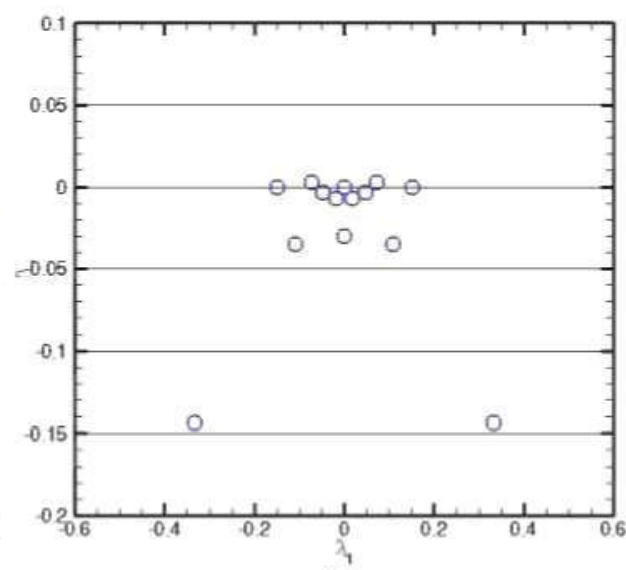

(b)
FIG. 17. Composite DMD analysis of the auxiliary channel flow databases, using composite $u^{\prime}\left(\vec{x}, t_{k}\right)-C_{f}\left(t_{k}\right)$ snapshots. Spectra, in (a), for standard and for actuated case, in (b).

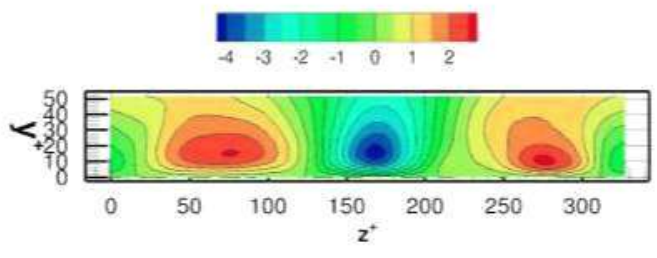

(a)

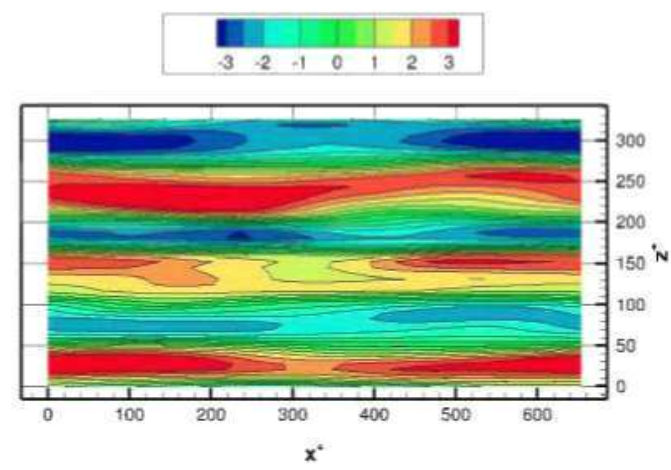

(b)

FIG. 18. Composite DMD analysis of the standard channel flow database, using composite $u^{\prime}\left(\vec{x}, t_{k}\right)-C_{f}\left(t_{k}\right)$ snapshots. Reconstructed flow field using Eq. $(6)$, with $n_{r}=5$. (a) Flow averaged over time and the $x$-direction; (b) instantaneous field at $y^{+} \approx 15$. Fields nondimensionalized by $u_{\tau}=0.04233$. 


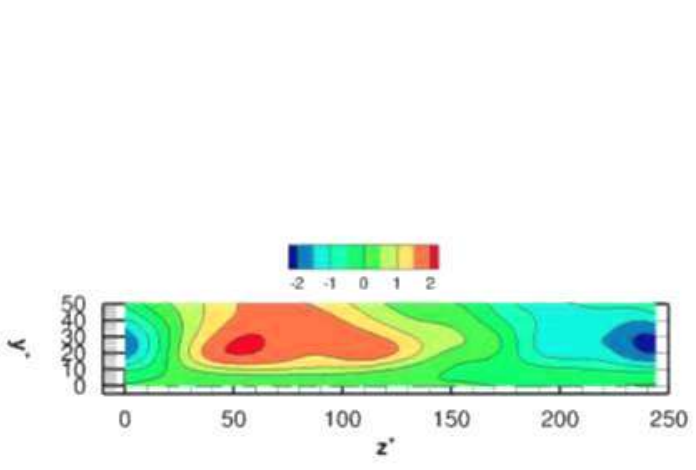

(a)

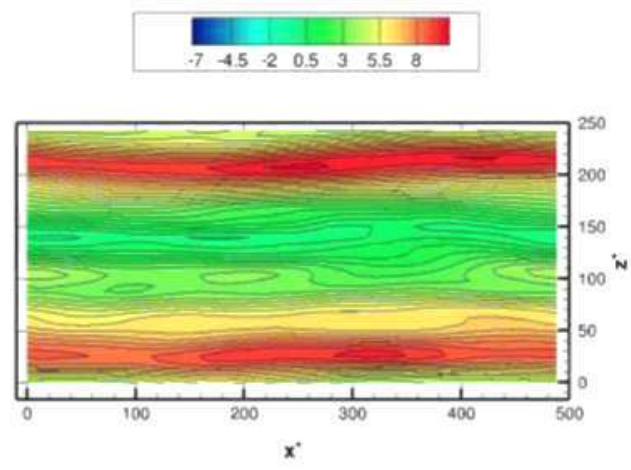

(b)

FIG. 19. Composite DMD analysis of the standard channel flow database, using composite $u^{\prime}\left(\vec{x}, t_{k}\right)-C_{f}\left(t_{k}\right)$ snapshots. Reconstructed flow field using Eq. $(6)$, with $n_{r}=3$. (a) Flow averaged over time and the $x$-direction; (b) instantaneous field at $y^{+} \approx 15$. Fields nondimensionalized by $u_{\tau}=0.03136$.

allows us to identify a few modes that replicate relevant features of the physical underlying process.

\section{REFERENCES}

${ }^{1}$ Post-Processing of Numerical and Experimental Data, LS 2008-01, edited by P. Millan and M. L. Riethmuller (von Karman Institute for Fluid Dynamics, Sint-Genesius-Rode, Belgium, 2008).

${ }^{2}$ Recent Advances in Particle Image Velocimetry, LS 2009-01, edited by F. Scarano and M. L. Riethmuller (von Karman Institute for Fluid Dynamics, Sint-GenesiusRode, Belgium, 2009).

${ }^{3}$ Large Eddy Simulation and Related Techniques, LS 2010-04, edited by U. Piomelli, C. Benocci, and J. P. A. J. van Beeck (von Karman Institute for Fluid Dynamics, Sint-Genesius-Rode, Belgium, 2010).

${ }^{4}$ ERCOFTAC Direct and Large-Eddy Simulation X, edited by D. G. E. Grigoriadis, B. J. Geurts, H. Kuerten, J. Fröhlich, and V. Armenio (Springer, 2018).

5. L. Lumley, Stochastic Tools in Turbulence (Academic Press, 1970).

${ }^{6} \mathrm{~L}$. Sirovich, "Turbulence and the dynamics of coherent structures," Q. Appl. Math. 45(3), 561-590 (1987).

${ }^{7}$ G. Berkooz, P. Holmes, and J. L. Lumley, "The proper orthogonal decomposition in the analysis of turbulent flows," Annu. Rev. Fluid Mech 25(1), 539-575 (1993).

${ }^{8} \mathrm{~S}$. Volkwein, Proper Orthogonal Decomposition: Theory and Reduced-Order Modelling: Lecture Notes, 2013.

${ }^{9}$ C. W. Rowley, I. Mezić, S. Bagheri, P. Schlatter, and D. S. Henningson, "Spectral analysis of nonlinear flows," J. Fluid Mech. 641, 115-127 (2009).

${ }^{10} \mathrm{P}$. J. Schmid, "Dynamic mode decomposition of numerical and experimental data," I. Fluid Mech. 656, 5-28 (2010).

${ }^{11}$ E. Ferrer, J. de Vicente, and E. Valero, "Low cost 3D global instability analysis and flow sensitivity based on dynamic mode decomposition and high-order numerical tools," Int. ). Numer. Methods Fluids 76(3), 169-184 (2014).

${ }^{12}$ N. E. Huang, Z. Shen, S. R. Long, M. C. Wu, H. H. Shih, Q. Zheng, N.-C. Yen, C. C. Tung, and H. H. Liu, "The empirical mode decomposition and the Hilbert spectrum for nonlinear and non-stationary time series analysis," Proc. R. Soc. London, Ser. A 454(1971), 903-995 (1998).

${ }^{13} \mathrm{~L}$. Agostini and M. A. Leschziner, "On the influence of outer large-scale structures on near-wall turbulence in channel flow," Phys. Fluids 26(7), 075107 (2014).

${ }^{14}$ A. Altuntas, L. Davidson, and S. H. Peng, "A new approximation to modulationeffect analysis based on empirical mode decomposition," Phys. Fluids 31(2), $025117(2019)$

${ }^{15}$ P. Moin and R. D. Moser, "Characteristic-eddy decomposition of turbulence in a channel," I. Fluid Meith. 200, 471-509 (1989).
${ }^{16}$ N. Aubry, P. Holmes, J. L. Lumley, and E. Stone, "The dynamics of coherent structures in the wall region of the wall boundary layer," H. Fuid Mech. 192. 115-173 (1988).

${ }^{17}$ D. Rempfer and H. F. Fasel, "Evolution of three-dimensional coherent structures in a flat-plate boundary layer," I. Fluid Me h. 260, 351-375 (1994).

${ }^{18} \mathrm{~B}$. Podvin, "On the adequacy of the ten-dimensional model for the wall layer," Phys. Fluids 13(1), 210-224 (2001).

${ }^{19}$ B. Podvin and J. L. Lumley, "A low-dimensional approach for the minimal flow unit," I. Fluid Mech. 362, 121-155 (1998).

${ }^{20} \mathrm{~B}$. Podvin, "A proper-orthogonal-decomposition-based model for the wall layer of a turbulent channel flow," Phys. Fluids 21(1), 015111 (2009).

${ }^{21} \mathrm{~F}$. Waleffe, "On a self-sustaining process in shear flows," Phys. Fluids $9(4)$, 883-900 (1997).

${ }^{22}$ J. Moehlis, H. Faisst, and B. Eckhardt, " A low-dimensional model for turbulent shear flows," New J. Phys, 6,56 (2004).

${ }^{23}$ M. Lagha and P. Manneville, "Modeling transitional plane Couette flow," Eur. Phys. I. B 58(4), 433-447 (2007).

${ }^{24}$ M. Lagha, "A comprehensible low-order model for wall turbulence dynamics," Pliys. Fluiùs 26(8), 085111 (2014).

${ }^{25} \mathrm{M}$. Lagha, J. Kim, J. D. Eldredge, and X. Zhong, "A numerical study of compressible turbulent boundary layers," Phys. Fluids 23(1), 015106 (2011).

${ }^{26} \mathrm{M}$. Lagha, J. Kim, J. D. Eldredge, and X. Zhong, "Near-wall dynamics of compressible boundary layers," Phys. Fluids 23(6), 065109 (2011).

${ }^{27}$ J. N. Kutz, S. L. Brunton, B. W. Brunton, and J. L. Proctor, Dynamic Mode Decomposition: Data-Driven Modeling of Complex Systems (Society for Industrial and Applied Mathematics, USA, 2016).

${ }^{28}$ I. Mezić, "Analysis of fluid flows via spectral properties of the Koopman operator," Annu. Rev. Fluid Mceh. 45(1), 357-378 (2013).

${ }^{29} \mathrm{~S}$. Bagheri, "Koopman-mode decomposition of the cylinder wake," T. Fhuid Mech. 726, 596-623 (2013).

${ }^{30}$ K. Taira, S. L. Brunton, S. T. M. Dawson, C. W. Rowley, T. Colonius, B. J. McKeon, O. T. Schmidt, S. Gordeyev, V. Theofilis, and L. S. Ukeiley, "Modal analysis of fluid flows: An overview," A1AA T.55(12), 4013-4041 (2017).

${ }^{31}$ K. K. Chen, J. H. Tu, and C. W. Rowley, "Variants of dynamic mode decomposition: Boundary condition, Koopman, and Fourier analyses," I. Nonlinear Sci. 22(6), 887-915 (2012).

${ }^{32}$ A. Towne, O. T. Schmidt, and T. Colonius, "Spectral proper orthogonal decomposition and its relationship to dynamic mode decomposition and resolvent analysis," T. Fluid Mech. 847, 821-867 (2018).

${ }^{33}$ S. Derebail Muralidhar, B. Podvin, L. Mathelin, and Y. Fraigneau, "Spatiotemporal proper orthogonal decomposition of turbulent channel flow," |. Fluid Mech. 864, 614-639 (2019). 
${ }^{34}$ D. Duke, J. Soria, and D. Honnery, "An error analysis of the dynamic mode decomposition," Exp. Fluids 52(2), 529-542 (2012).

${ }^{35}$ P. J. Schmid, D. Violato, and F. Scarano, "Decomposition of time-resolved tomographic PIV," Exp. Fluids 52(6), 1567-1579 (2012).

${ }^{36} \mathrm{P}$. J. Schmid, "Application of the dynamic mode decomposition to experimental data," Exp. Fluids 50(6), 1123-1130 (2011).

${ }^{37}$ P. J. Schmid, L. Li, M. P. Juniper, and O. Pust, "Applications of the dynamic mode decomposition," Thent Comput. Fluid Dyn. 25(1), 249-259 (2011).

${ }^{38}$ S. Le Clainche, J. M. Vega, and J. Soria, "Higher order dynamic mode decomposition of noisy experimental data: The flow structure of a zero-net-mass-flux jet," Exp. Therm. Fluid Sui 88(Suppl. C), 336-353 (2017).

${ }^{39} \mathrm{~T}$. Sayadi and P. J. Schmid, "Parallel data-driven decomposition algorithm for large-scale datasets: With application to transitional boundary layers," Theor: Comput. Fluid Dyn. 30(5), 415-428 (2016).

${ }^{40}$ T. Sayadi, P. J. Schmid, J. W. Nichols, and P. Moin, "Reduced-order representation of near-wall structures in the late transitional boundary layer," |. Fluid Mech. 748, 278-301 (2014).

${ }^{41}$ A. Cassinelli; M. de Giovanetti, and Y. Hwang, "Streak instability in near-wall turbulence revisited," T. Turhul. 18(5), 443-464 (2017).

${ }^{42}$ T. Grenga, J. F. MacArt, and M. E. Mueller, "Dynamic mode decomposition of a direct numerical simulation of a turbulent premixed planar jet flame: Convergence of the modes," Combust. Theory Modell. 22(4),795-811 (2018).

${ }^{43} \mathrm{~S}$. Le Clainche and J. M. Vega, "Higher order dynamic mode decomposition," SIAM J. Appl. Dyn. Syst. 16(2), 882-925 (2017).

${ }^{44} \mathrm{~S}$. Le Clainche and J. M. Vega, "Analyzing nonlinear dynamics via data-driven dynamic mode decomposition-like methods," Complexity 2018, 1.

${ }^{45} \mathrm{~S}$. Le Clainche, Z. H. Han, and E. Ferrer, "An alternative method to study crossflow instabilities based on high order dynamic mode decomposition," Phys. Fluids 31(9), 094101 (2019).

${ }^{46} \mathrm{~S}$. Le Clainche and E. Ferrer, "A reduced order model to predict transient flows around straight bladed vertical axis wind turbines," Energies 11(3), 566 (2018).

${ }^{47}$ M. R. Jovanovic, P. J. Schmid, and J. W. Nichols, "Sparsity-promoting dynamic mode decomposition," Phys. Fluids 26(2), 024103 (2014).

${ }^{48}$ F. Guéniat, L. Mathelin, and L. R. Pastur, "A dynamic mode decomposition approach for large and arbitrarily sampled systems," Phys. Fluids 27(2), 025113 (2015).

${ }^{49}$ T. Bui-Thanh, M. Damodaran, and K. E. Willcox, "Aerodynamic data reconstruction and inverse design using proper orthogonal decomposition," AIAA F. 42(8), 1505-1516 (2004).
${ }^{50} \mathrm{~T}$. Braconnier, M. Ferrier, J.-C. Jouhaud, M. Montagnac, and P. Sagaut, "Towards an adaptive POD/SVD surrogate model for aeronautic design," Comput. Fluids 40(1), 195-209 (2011).

${ }^{51} \mathrm{P}$. Luchini and M. Quadrio, "A low-cost parallel implementation of direct numerical simulation of wall turbulence," I. Comput. Phys. 211(2), 551-571 (2006).

${ }^{52} \mathrm{C}$. W. Rowley and S. T. M. Dawson, "Model reduction for flow analysis and control," Annu. Rev. Fluid Mech. 49(1), 387-417 (2017).

${ }^{53} \mathrm{~K}$. Fukagata, K. Iwamoto, and N. Kasagi, "Contribution of Reynolds stress distribution to the skin friction in wall-bounded flows," Phys. Fluids 14(11), L73 (2002).

${ }^{54} \mathrm{C}$. Viotti, M. Quadrio, and P. Luchini, "Streamwise oscillation of spanwise velocity at the wall of a channel for turbulent drag reduction," Phys. Fluids 21(11), 115109 (2009).

${ }^{55}$ J. Kim, P. Moin, and R. Moser, "Turbulence statistics in fully developed channel flow at low Reynolds number," f. Fluid Mech. 177, 133-166 (1987).

${ }^{56} \mathrm{M}$. Quadrio and P. Ricco, "Critical assessment of turbulent drag reduction through spanwise wall oscillations," I. Fluid Mech. 521, 251-271 (2004).

${ }^{57} \mathrm{M}$. Quadrio, P. Ricco, and C. Viotti, "Streamwise-traveling waves of spanwise wall velocity for turbulent drag reduction," I. Fluid Mech. 627, 161-178 (2009).

${ }^{58}$ S. Ghebali, S. I. Chernyshenko, and M. Leschziner, "Can large-scale oblique undulations on a solid wall reduce the turbulent drag?," Phys. Fluids 29, 105102 (2017).

${ }^{59} \mathrm{G}$. E. Karniadakis and K.-S. Choi, "Mechanisms on transverse motions in turbulent wall flows," Annu. Rey. Fluid Mech. 35(1), 45-62 (2003).

${ }^{60}$ Y. Saad, Numerical Methods for Large Eigenvalue Problems (Manchester University Press, Manchester, 1992).

${ }^{61} \mathrm{~J}$. Kou and W. Zhang, "An improved criterion to select dominant modes from dynamic mode decomposition," Eur. I. Mech.: B/Fluids 62, 109-129 (2017).

${ }^{62}$ M. Quadrio, B. Frohnapfel, and Y. Hasegawa, "Does the choice of the forcing term affect flow statistics in DNS of turbulent channel flow?," Eur. T. Mech.. B/Fluids 55, 286-293 (2016), vortical structures and wall turbulence.

${ }^{63}$ S. Pope, Turbulent Flows, 2nded. (CUP, 2014).

${ }^{64} \mathrm{~J}$. Jiménez and A. Pinelli, "The autonomous cycle of near-wall turbulence," T. Fluid Mech. 389, 335-359 (1999).

${ }^{65} \mathrm{~J}$. Kim and F. Hussain, "Propagation velocity of perturbations in turbulent channel flow," Phys. Fluids A 5(3), 695-706 (1993).

${ }^{66}$ W. Schoppa and F. Hussain, "Coherent structure generation in near-wall turbulence," F. Fluid Mech. 453, 57-108 (2002). 